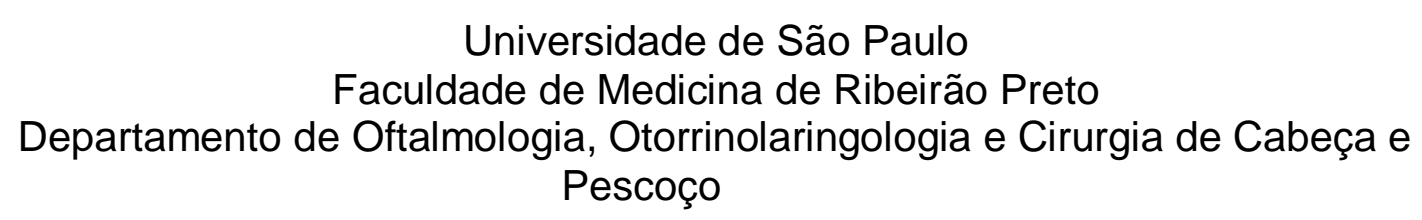

DENISE SILVA MATURO

\title{
Nasalância em indivíduos com deformidades dentofaciais e a influência da cirurgia ortognática
}




\section{Nasalância em indivíduos com deformidades dentofaciais e a influência da cirurgia ortognática}

\section{Versão original}

Dissertação

Departamento

Otorrinolaringologia e Pescoço da Faculdade de Medicina de Ribeirão Preto da Universidade de São Paulo, para obtenção do Título de Mestre em Ciências Médicas.

Área de concentração: Morfofisiologia de Estruturas Faciais

Orientadora: Profa. Dra. Luciana Vitaliano Voi Trawitzki 
Autorizo a reprodução e divulgação total ou parcial deste trabalho, por qualquer meio convencional ou eletrônico, para fins de estudo e pesquisa, desde que citada a fonte.

Maturo, Denise Silva

Nasalância em indivíduos com deformidades dentofaciais e a influência da cirurgia ortognática. Ribeirão Preto, 2017.

74 p. : il. ; $30 \mathrm{~cm}$

Dissertação de Mestrado, apresentada à Faculdade de Medicina de Ribeirão Preto/USP. Área de concentração: Morfofisiologia de Estruturas Faciais.

Orientador: Trawitzki, Luciana Vitaliano Voi

1. Nasalância. 2. Deformidades dentofacias. 3. Cirurgia ortognática. 4. Nasalidade de fala. 5. Nasometria. 6. Nasômetro. 
Maturo DS. Nasalância em indivíduos com deformidades dentofaciais e a influência da cirurgia ortognática [dissertação]. Ribeirão Preto: Universidade de São Paulo, Faculdade de Medicina de Ribeirão Preto; 2017.

Aprovado em:

Banca Examinadora

Profa. Dra.

Instituição:

Julgamento:

Profa. Dra.

Instituição:

Julgamento:

Profa. Dra.

Instituição:

Julgamento: 


\section{AGRADECIMENTOS}

Aos meus pais, José Roberto Maturo e Lidionete Silva Maturo, por todo apoio, dedicação e por tudo que me proporcionaram até hoje. Sem vocês não teria chegado até aqui. Muito obrigada!

À Profa. Dra. Luciana Vitaliano Voi Trawitzki, pela disponibilidade, apoio, orientação e por todo ensinamento transmitido desde a graduação.

À Profa. Dra. Aline Epiphanio Wolf, pela correção e sugestões propostas que enriqueceram este trabalho.

À Profa. Dra. Jeniffer de Cássia Rillo Dutka, pela atenção e correção deste trabalho.

À Profa. Dra. Lilian Neto Aguiar Ricz por disponibilizar o Laboratório de Investigação de Voz e Fala e o equipamento nasômetro.

À Luciana Gonçalves de Aguiar Campanini e à Maria Cecília Onofre pelo apoio, atenção e esclarecimentos no decorrer da pós-graduação.

Ao Dr. Denny Marcos Garcia pela contribuição neste trabalho, com seus conhecimentos na área de estatística.

Aos voluntários e pacientes que participaram deste trabalho.

À equipe do LISE e do CIEDEF pelo apoio na coleta dos dados, parceria e amizade durante esses anos.

Às amigas e amigos da pós-graduação, pela parceria e companheirismo durante esses anos de muito trabalho e aprendizado.

Ao Departamento de Oftalmologia, Otorrinolaringologia e Cirurgia de Cabeça e Pescoço da Faculdade de Medicina de Ribeirão Preto, pela oportunidade de realização do mestrado.

À Coordenação de Aperfeiçoamento de Pessoal de Nível Superior - CAPES, pela concessão da bolsa de mestrado.

À Fundação de Amparo a Pesquisa do Estado de São Paulo - FAPESP, pelo auxílio à pesquisa para aquisição do nasômetro. 


\section{RESUMO}

Maturo DS. Nasalância em indivíduos com deformidades dentofaciais e a influência da cirurgia ortognática [dissertação]. Ribeirão Preto: Universidade de São Paulo, Faculdade de Medicina de Ribeirão Preto; 2017.

A nasalidade da fala sofre influência de fatores como tamanho e formato da cavidade oral e da configuração da cavidade nasal. Nas deformidades dentofaciais são encontradas alterações no crescimento dos ossos da mandíbula e/ou maxila, estruturas diretamente relacionadas a estas cavidades. $O$ objetivo deste estudo foi analisar a influência da deformidade dentofacial e o efeito da cirurgia ortognática nos escores de nasalância, em um acompanhamento de 6 meses de pós-operatório. Para o desenvolvimento deste estudo foram triados 146 indivíduos, de ambos os sexos, com idade superior a 18 anos, alfabetizados, sem distinção de raça e nível socioeconômico. Foram selecionados 91 indivíduos, sendo $53 \mathrm{com}$ deformidades dentofaciais (23 classe II, idade média 27,4 anos; e 31 classe III, idade média 27,2 anos) e 37 sem deformidade dentofacial (grupo controle, idade média 25,3 anos). Os sujeitos foram submetidos inicialmente a uma entrevista e a uma avaliação miofuncional orofacial, em seguida, foi avaliado o fluxo aéreo nasal, por meio do espelho Milimetrado de Altmann $\circledast$, e realizada a nasometria utilizando o Nasômetro II modelo 6400 (KayPENTAX, New Jersey - USA), durante a leitura de 10 frases padronizadas, do português brasileiro. Nos sujeitos com deformidades dentofaciais estes procedimentos foram repetidos em 3 períodos distintos do pós-operatório (P.O. I, II e III). Para a análise estatística foram utilizados os testes ANOVA two-way $(p<0,05)$, para analisar o efeito da cirurgia nos escores de nasalância, e te Student $(p<0,05)$, para identificar em qual período ocorreu. No período pré-operatório os valores médios e os desvios-padrão dos escores de nasalância dos indivíduos controles e com deformidades dentofaciais classe II e III foram, respectivamente, $48.1 \%$ (5.9), $48.9 \%$ (6.7) e $45.4 \%$ (9.7) para o texto nasal e $12.0 \%$ (5.3), $14.9 \%$ (7.0) e $10.6 \%$ (5.4) para o texto oral, valores dentro dos padrões de normalidade para indivíduos brasileiros; a análise desses dados não mostrou diferença significativa entre os grupos. Quanto ao efeito da cirurgia ortognática nos escores de nasalância houve diferença estatisticamente significante para os fatores tempo-grupo no texto 
oral, diferença evidenciada entre os períodos pré-operatório e P.O. III. Diante dos achados, pode-se concluir que o tipo de deformidade dentofacial pareceu não influenciar os escores de nasalância, já a cirurgia ortognática provocou efeito nesses escores, dentro de um período de 6 meses, porém sem alterar as características da nasalidade da fala, visto que os escores de nasalância se mantiveram dentro dos padrões de normalidade.

Palavras-chave: Nasalância. Deformidades dentofaciais. Cirurgia ortognática. Nasalidade de fala. Nasometria. Nasômetro. 


\begin{abstract}
Maturo DS. Nasalance in subjects with dentofacial deformities and the influence of orthognathic surgery [dissertation]. Ribeirão Preto: Universidade de São Paulo, Faculdade de Medicina de Ribeirão Preto; 2017.
\end{abstract}

Factors such as size and shape of the oral cavity and the nasal cavity configuration may influence nasalance scores. In dentofacial deformities the abnormal growth of jaw and maxillary bones is directly related with changes in these cavities. The aim of this study was to analyze the influence of dentofacial deformity and the effect of orthognathic surgery on nasalance scores, during a 6-month post-operative followup. In order to develop this study, 146 individuals of both sexes, over 18 years older, literate, without distinction of race and socioeconomic status, were screened. Ninetyone individuals were selected, which 53 had dentofacial deformities (23 class II, mean age 27.4 years, and 31 class III, mean age 27.2 years) and 37 without dentofacial deformity (control group, mean age 25.3 years). The subjects were submitted to an interview and an orofacial myofunctional evaluation, then the nasal airflow was evaluated through the Altmann® graded mirror, and the nasometry was acquired with Nasometer II model 6400 (KayPENTAX, New Jersey - USA ) device, based on reading of 10 sentences standardized, from Brazilian Portuguese. In subjects with dentofacial deformities these procedures were repeated in 3 different postoperative periods (P.O. I, II and III). To analyze the surgery effect on nasalance scores it was used ANOVA two-way test $(p<0.05)$ and Student's $t$ test $(p<0.05)$ was used to identify which period it occurred. In the preoperative period, the mean values and the standard deviations of the nasalance scores of the control group and groups with class II and III dentofacial deformities were, respectively, 48.1\% (5.9), 48.9\% (6.7) and 45.4\% (9.7) for the nasal text and 12.0\% (5.3), 14.9\% (7.0) and 10.6\% (5.4) for the oral text, values within normality standards for Brazilian individuals; analyzing these data no significant difference between the groups were found. About effect of orthognathic surgery on nasalance scores it was found a significant difference in time-group factor for the oral text and comparing the mean nasalance scores in the different periods, the difference was evidenced between the preoperative and PO III periods. Considering the findings, it can be concluded that the type of dentofacial 
deformity did not appear to influence the nasalance scores, whereas the orthognathic surgery had an effect on these scores within a period of 6 months, but did not changing the nasality characteristics of the speech, because groups had scores within the normal range.

Keywords: Nasalance. Dentofacial deformities. Orthognathic surgery. Speech nasality. Nasometry. Nasometer. 


\section{LISTA DE FIGURAS}

Figura 1 - Fluxograma de distribuição da amostra

Figura 2 - Espelho Milimetrado de Altmann - Pró-Fono®

Figura 3 - Instrumento para mensuração da nasalância 


\section{LISTA DE TABELAS}

Tabela 1 - Distribuição da amostra quanto aos grupos, número de sujeitos, sexo e idade, no período pré-operatório

Tabela 2 - Distribuição da amostra em porcentagem (\%), quanto aos grupos (GC, Gll e GIII) e estado de origem

Tabela 3 - Variação do período de avaliação, em dias

Tabela 4 - Distribuição da amostra quanto aos grupos (GIl e GIII) e procedimentos / técnicas cirúrgicas

Tabela 5 - Porcentagem de sujeitos que realizaram fonoterapia de acordo com os grupos (Gll e GllI) e procedimentos fonoterápicos no período pós-operatório

Tabela 6 - Valores médios e desvio padrão de nasalância (\%) dos grupos Controle, Classe II e Classe III no pré-operatório

Tabela 7 - Influência e interação dos fatores grupo e sexo nos valores médios de nasalância para os textos nasal e oral

Tabela 8 - Relação da proporção dos sexos nos grupos

Tabela 9 - Influência e interação dos fatores grupo e tempo nos valores médios de nasalância para os textos nasal e oral

Tabela 10 Valores médios e desvios padrão de nasalância (\%) dos grupos Classe II e Classe III, nos diferentes períodos de avaliação nasométrica 


\section{LISTA DE ABREVIATURAS E SIGLAS}

$\begin{array}{ll}\text { AMIOFE } & \text { Avaliação Miofuncional Orofacial com Escores } \\ \text { ANOVA } & \text { Teste estatístico de Análise de Variância } \\ \text { CIEDEF } & \text { Centro Integrado de Estudos das Deformidades da Face } \\ \text { EVF } & \text { Esfíncter Velofaríngeo } \\ \text { FVF } & \text { Função Velofaríngea } \\ \text { GC } & \text { Grupo Controle } \\ \text { GII } & \text { Grupo deformidade dentofacial classe II } \\ \text { GIII } & \text { Grupo deformidade dentofacial classe III } \\ \text { LISE } & \text { Laboratório de Investigação do Sistema Estomatognático } \\ \text { LIVF } & \text { Laboratório de Investigação da Voz e Fala } \\ \text { P.O. I } & \text { Pós-operatório I } \\ \text { P.O. II } & \text { Pós-operatório II } \\ \text { P.O. III } & \text { Pós-operatório III }\end{array}$




\section{SUMÁRIO}

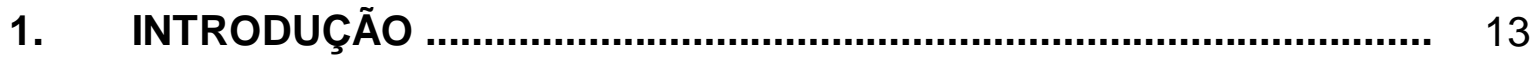

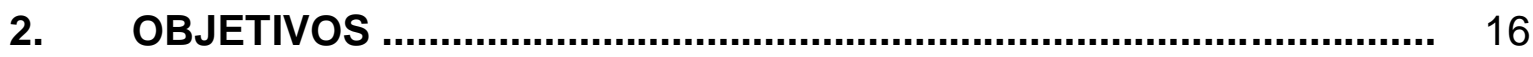

2.1 Objetivos específicos …................................................................. 17

3. REVISÃO DE LITERATURA ….................................................. 18

3.1 Ressonância e Mecanismo Velofaríngeo ........................................ 19

3.2 Deformidade dentofacial e cirurgia ortognática ............................... 20

3.3 Nasalidade e nasalância da fala ...................................................... 23

3.4 Nasalância e cirurgia ortognática ................................................ 26

4. MATERIAIS E MÉTODOS …........................................................ 30

4.1 Considerações Éticas .................................................................. 31

4.2 Tipo de Estudo ............................................................................... 31

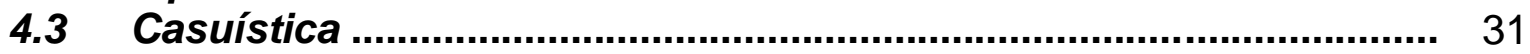

4.3.1 Caracterização da amostra ........................................................... 31

4.3.2 Critérios de inclusão …….................................................................. 33

4.3.3 Critérios de exclusão ……................................................................. 34

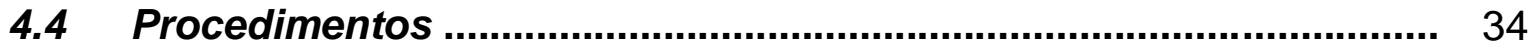

4.4.1 Avaliação clínica fonoaudiológica ................................................... 35

4.4.2 Nasometria ............................................................................. 36

4.4.3 Tratamentos realizados ................................................................. 37

$4.5 \quad$ Análises estatísticas ................................................................... 39

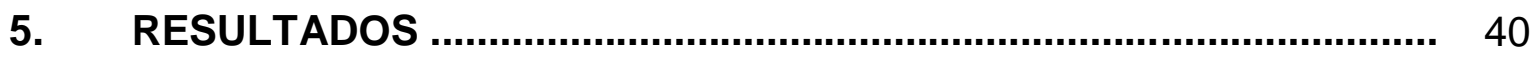

6. DISCUSSÃO ................................................................................. 44

7. CONCLUSÕES ..................................................................... 52

REFERÊNCIAS BIBLIOGRÁFICAS ............................................... 54

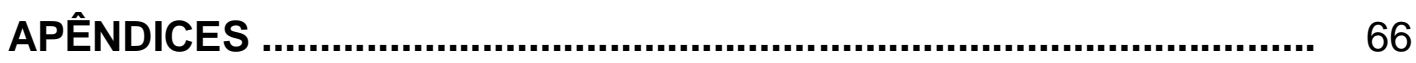

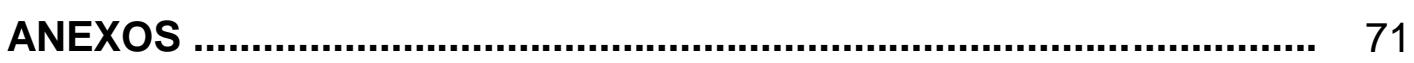


1. INTRODUÇÃO 


\section{INTRODUÇÃO}

A fala, um processo complexo que envolve o sistema neuromuscular, resulta da coordenação eficaz entre vários subsistemas físicos que incluem a respiração, a fonação, a articulação e a ressonância (Kummer, 2013). O volume do fluxo e pressão do ar, além da ressonância, também são fundamentais para a sua correta produção (Marchesan, 2004).

A ressonância da fala sofre influência do funcionamento do esfíncter velofaríngeo (EVF), do tamanho e formato da cavidade oral e da configuração da cavidade nasal (Kummer, 2013). Portanto, para seu equilíbrio, o funcionamento adequado do EVF e as principais cavidades de ressonância (faringe, cavidade oral e nasal) devem estar íntegras.

De acordo com as características físico-estruturais, podemos dizer que indivíduos com deformidade dentofacial tem uma maior probabilidade de ter alterações de ressonância, visto que há uma alteração de crescimentos dos ossos, maxila e mandíbula, diretamente relacionados com as cavidades nasal e oral.

A maneira mais comum e simples de avaliar a ressonância ou nasalidade da fala é por julgamento perceptivo-auditivo (Genaro; Yamashita; Trindade, 2009). Porém, muitos autores apontam a importância da avaliação instrumental, por ser um método objetivo e não causar discordâncias nos resultados (Trindade; Genaro; Dalston, 1997; Van Lierde; Wuyts; Bonte, 2007; Trindade; Yamashita; Gonçalves, 2007; Brunnegård; Lohmander; Van Doorn, 2012). O instrumento mais utilizado na literatura para avaliar a nasalidade de fala é o nasômetro, que fornece dados quantitativos, chamados escores de nasalância.

A nasalância é o correlato acústico da nasalidade, portanto, pode variar de acordo com fatores como tamanho e volume da cavidade nasal (Ko et al., 1999; Pegoraro-Krook et al., 2006; Birkent et al., 2009; Kim et al., 2013).

A cirurgia ortognática, procedimento muito utilizado para correção das deformidades dentofaciais, modifica as vias aéreas superiores (Kim J-S et al., 2010; Jakobsone; Stenvik; Espeland, 2011; Mattos et al., 2011; Park et al., 2012; Williams et al., 2013; Chang et al., 2015; Al-Moraissi et al., 2015; Marşan et al., 2015; Karabekmez; Kleinheinz; Jung, 2015; Kochar et al., 2016), portanto, é outro fator que pode trazer mudanças nos escores de nasalância (Haapanen et al., 1997; Ward et 
al., 2002; Trindade et al., 2003; Van Lierde et al., 2003; Zemann et al., 2006; Smedberg; Neovius; Lohmander, 2014).

Chanchareonssok, Samman N e Whitehill (2006) e Hassan, Naini e Gill (2007) realizaram trabalhos de revisão da literatura sobre os efeitos da cirurgia ortognática nas características da fala e concluíram que os resultados não são claros e por vezes, contraditórios. Portanto, torna-se importante caracterizar a nasalância de indivíduos com diferentes deformidades dentofaciais, no pré e pósoperatório de cirurgia ortognática, para entender melhor os efeitos desta sobre a fala e investigar se as mudanças são permanentes ou transitórias. 


\section{OBJETIVOS}

O propósito deste estudo foi analisar a influência da deformidade dentofacial nos escores de nasalância, assim como da cirurgia ortognática, em um acompanhamento de 6 meses pós-operatório.

\subsection{Objetivos especificos}

Verificar se:

- Existe diferença nos escores de nasalância entre indivíduos com deformidades dentofaciais classe II e classe III, considerando a discrepância maxilomandibular de cada deformidade;

- A cirurgia ortognática, envolvendo a maxila associada ou não à cirurgia de mandíbula, influencia nos escores de nasalância desses indivíduos, e em qual período, considerando um acompanhamento de 6 meses pós-operatório. 


\section{REVISÃO DE LITERATURA}

\subsection{Ressonância e Mecanismo Velofaríngeo}

A ressonância da fala pode ser classificada como normal ou adequada, quando há o uso equilibrado das cavidades de ressonância; hipernasal, quando há uso excessivo da cavidade nasal; hiponasal, quando o uso cavidade nasal é insuficiente; laríngea, quando há uso excessivo da laringe; faríngea, caracterizada por tensão na faringe; e laringofaríngea, quando há tensão da laringe e faringe (Behlau et al. 2001; Kummer, 2013).

Um dos fatores que influencia a ressonância da fala é o funcionamento do $E V F$, ou seja, é necessário que haja o movimento sincronizado das estruturas do mecanismo velofaríngeo (elevação e posteriorização do palato mole, mesialização das paredes laterais e anteriorização da parede posterior da faringe) (PegoraroKrook et al., 2004), para que a distribuição do fluxo aéreo expiratório e das vibrações acústicas para a cavidade oral e nasal sejam adequadas (Kent, 1997; Camargo; Rodrigues; Avelar, 2001; Pegoraro-Krook et al., 2004; Kummer, 2013).

O funcionamento normal da função velofaríngea (FVF) é determinado por componentes como anatomia, fisiologia e aprendizado. Portanto, além de ter as estruturas íntegras, o falante deve aprender a usar o EVF corretamente na tentativa de produzir os sons orais da fala (Kummer, 2011). Quando há uma inadequação no fechamento do mecanismo velofaríngeo durante a produção de sons orais, as ondas acústicas adentram a cavidade nasal de forma indesejada, produzindo alteração da ressonância, ou hipernasalidade (Baken, 1997; Genaro; Fukushiro; Suguimoto, 2007; Kummer, 2013). Essa inadequação é conhecida, genericamente, como disfunção velofaríngea.

Existem dois tipos de disfunção velofaríngea, diferenciados de acordo com sua origem: incompetência velofaríngea, de origem neurofisiológica (hipotonia generalizada, condições neurológicas causando disartria ou apraxia e danos nos nervos cranianos) e insuficiência velofaríngea, de origem estrutural ou anatômica (fissuras de palato, faringe profunda, atrofia ou irregularidade de adenoides, hipertrofia de tonsilas, adenoidectomia, tonsilectomia, tumores da cavidade oral e avanço de maxila) (Kummer, 2011). De acordo com Kummer (2013) a ressonância 
ainda pode ser afetada pelo comprimento e volume da faringe, pelo tamanho e formato da cavidade oral e pela configuração da cavidade nasal.

Alguns autores relatam que sujeitos com deformidades dentofaciais apresentam variações das dimensões faríngeas e das vias aéreas superiores, quando comparados entre si e a sujeitos sem deformidade (Kirjavainen; Kirjavainen, 2007; Grauer et al., 2009; Kim Y-J et al., 2010; El; Palamo, 2011, 2013; Hong et al., 2011; Martin; Muelas; Viñas, 2011; Alves Jr et al., 2012; Castro-Silva et al., 2015).

Portanto, diante do exposto, podemos supor que sujeitos com deformidades dentofaciais são predispostos a terem alterações de ressonância da fala.

\subsection{Deformidade dentofacial e cirurgia ortognática}

Alterações do padrão de crescimento da face, devido a fatores genéticos (Felício, 1999; Trawitzki et al., 2000; Tucker, 2000; Tanigute, 2005) e/ou alterações nas funções do sistema estomatognático, podem modificar a morfologia e oclusão dentária (Felício, 1999; Tanigute, 2005; Felício 2009), favorecendo as alterações no desenvolvimento dentofacial, como as desproporções maxilomandibulares.

As deformidades dentofaciais são caracterizadas por alterações na relação maxilomandibular, proporcionando uma desarmonia craniofacial. A deformidade dentofacial classe II caracteriza-se por deficiência de crescimento da mandíbula e/ou pela protrusão maxilar, e a deformidade dentofacial classe III apresenta como característica o prognatismo (excesso de crescimento da mandíbula) e/ou a deficiência de crescimento da maxila. (Capelozza Filho, 2004; Proffit; White Jr, 1990).

Visto que a ressonância da fala sofre influência do tamanho e formato da cavidade oral e da configuração da cavidade nasal (Kummer, 2013) é esperado que sujeitos com deformidades dentofaciais apresentem alterações de ressonância da fala. Mason et al. (1980) apontam que a hipoplasia do espaço nasofaríngeo, comumente associado à deficiência de crescimento de maxila ou do terço médio da face, justifica a hiponasalidade encontrada em alguns sujeitos com deformidade dentofacial.

Contudo, a literatura é pouco clara neste aspecto, pois apresenta trabalhos em que o tipo de má-oclusão não influencia a largura da área faríngea (Freitas et al., 
2006; Alves et al., 2008). Todavia há também uma série de estudos mostrando que as dimensões faríngeas e das vias aéreas superiores variam de acordo com as deformidades dentofaciais (Kirjavainen; Kirjavainen, 2007; Grauer et al., 2009; Kim Y-J et al., 2010; Martin; Muelas; Viñas, 2011; Hong et al., 2011; Alves Jr et al., 2012; El; Palamo, 2011, 2013; Castro-Silva et al., 2015).

Grauer et al. (2009) relataram que o volume e o formato das vias aéreas diferem entre pacientes com diferentes deformidades dentofaciais, no sentido anteroposterior. Kim Y-J et al. (2010) e El e Palamo (2011) observaram que em pacientes com retrognatia o volume total das vias aéreas é menor quando comparado a sujeitos sem deformidades. Em 2013, El e Palamo confirmaram que sujeitos com deformidade dentofacial classe II, por retrognatia de mandíbula, apresentam menor volume das vias aéreas oro e nasofaríngea, principalmente quando comparados com sujeitos com deformidade dentofacial classe III, devido à prognatia.

A cirurgia ortognática, considerada padrão ouro para correção das discrepâncias da relação maxilomandibular, visa reestabelecer o equilíbrio craniofacial. As técnicas de osteotomias empregadas variam de acordo com a deformidade, podendo envolver a maxila, a mandíbula ou ambas as estruturas (Xavier; Ribeiro; Pedrosa-Junior, 2009).

A técnica indicada para correção da maxila é a Le Fort I, que possibilita a movimentação nos três planos espaciais, bem como a modificação do formato das arcadas dentárias e nivelamento dos arcos dentários (Ilg, 1999), permitindo então, o avanço ou segmentação da maxila (Xavier; Ribeiro; Pedrosa-Junior, 2009).

Para a correção da mandíbula as principais técnicas de osteotomias são vertical do ramo da mandíbula e sagital bilateral do ramo da mandíbula (Tucker, 2000).

A osteotomia vertical do ramo da mandíbula é utilizada, principalmente, para recuo mandibular em caso de prognatismo, para correção de laterodesvios e mais recentemente, para tratamento do desarranjo interno das articulações temporomandibulares (Ilg, 1999; Xavier; Ribeiro; Pedrosa-Junior, 2009). Normalmente, nessa técnica não se usa qualquer tipo de síntese óssea (fixação interna rígida) sendo, portanto, necessário o bloqueio maxilomandibular, no período de 3 a 6 semanas, para assegurar a consolidação da osteotomia (llg, 1999). 
A osteotomia sagital bilateral do ramo da mandíbula permite a movimentação anteroposterior do segmento, possibilitando seu avanço nos casos de retrognatismo, e seu recuo nos casos de prognatismo. Para o reposicionamento dos segmentos ósseos são utilizadas miniplacas e parafusos de titânio como fixação interna rígida (Ilg, 1999; Xavier; Ribeiro; Pedrosa-Junior, 2009).

Ainda para correção de deformidades dentofaciais em mandíbula, pode-se utilizar também a técnica da mentoplastia, geralmente em conjunto com outra osteotomia, para projeção ou redução do mento (de Freitas, 1999).

As osteotomias supracitadas podem ser realizadas isoladamente ou associadas. Quando associadas, recebem o nome de cirurgia combinada e são geralmente indicadas em casos de assimetrias importantes ou de deformidades dentofaciais severas (Xavier; Ribeiro; Pedrosa-Junior, 2009).

Além de restabelecer o equilíbrio entre a face e o crânio, a cirurgia ortognática visa favorecer a função mastigatória, a fonética, a respiração e a estética facial (Ribas et al., 2005). Alterações miofuncionais são notórias nas deformidades dentofaciais (Berretin-Felix; Jorge; Genaro, 2009; Trawitzki, 2009; Picinato-Pirola; Mello-Filho; Trawitzki, 2012; Nascimento, 2015) e mesmo após o tratamento cirúrgico algumas características musculares e das funções estomatognáticas permanecem alteradas (Berretin-Felix; Jorge; Genaro, 2009; Trawitzki, 2009). Por esse motivo, é de suma importância a atuação de uma equipe multiprofissional. $O$ trabalho fonoaudiológico, nesses casos, destina-se à adequação miofuncional orofacial e à conscientização do novo perfil facial (Marchesan; Bianchini, 1999; Trawitzki, 2009; Trawitzki; Borges; Grechi, 2016).

Vários estudos mostram que a cirurgia ortognática causa mudanças também nas vias aéreas superiores (Kim J-S et al., 2010; Jakobsone; Stenvik; Espeland, 2011; Mattos et al., 2011; Park et al., 2012; Williams et al., 2013; Chang et al., 2015; Al-Moraissi et al., 2015; Karabekmez; Kleinheinz; Jung, 2015; Marşan et al., 2015; Kochar et al., 2016). Al-Moraissi et al. (2015) relataram que a técnica cirúrgica também influencia na mudança do espaço aéreo faríngeo. Portanto, muitas implicações estão envolvidas neste tratamento cirúrgico, pois além das mudanças faciais que repercutem na vida pessoal e social do indivíduo (Ribas et al., 2005), podem ocorrer também mudanças com repercussão positiva ou negativa na respiração, na ressonância (Ward et al., 2002; Trindade et al., 2003; Niemeyer et al., 
2005; Chua et al., 2010; Pereira; Sell; Tuomainen, 2013) e na articulação da fala (Vallino 1990, Lee et al., 2002, Janulewicz et al., 2004, O’Gara; Wilson, 2007).

\subsection{Nasalidade e nasalância da fala}

A nasalidade é uma característica perceptivo-auditiva da ressonância, ou seja, corresponde à percepção subjetiva que um ouvinte tem da energia acústica nasal produzida durante a fala (Fletcher, 1976; Fletcher; Adams; Mccutcheon, 1989).

Facilmente identificada na produção de sons nasais, a nasalidade está presente em diferentes línguas e pode sofrer influência de fatores como os dialetos que a própria língua apresenta, devido aos efeitos fonológicos da variação linguística nas diferentes regiões do país (Seaver et al., 1991; Dalston; Neiman; GonzálezLanda, 1993; Annelin; Hallongren, 2006; Narece, 2007; Okalidou et al., 2011; Awan et al., 2015; Kim et al., 2016; Maturo, 2017). Outros fatores que podem influenciá-la são o sexo (Litzaw; Dalston, 1992; Suguimoto; Pegoraro-Krook, 1996; Rochet et al., 1998; Van Lierde et al., 2001; Di Ninno et al., 2001; Annelin; Hallongren, 2006; Mishima et al., 2008; Karakoc et al., 2013; Park et al., 2014; Kim et al., 2016) e a idade dos indivíduos (Trindade; Genaro; Dalston, 1997; Rochet et al., 1998; Di Ninno et al., 2001; Van Lierde et al., 2003; Annelin; Hallongren, 2006; Brunnegård; Van Doorn, 2009; Abou-Elsaad et al., 2012; Bettens et al., 2013; Lee; Browne, 2013; ElKassabi et al., 2014; Park et al., 2014; Ha; Cho, 2015), o tamanho e volume da cavidade nasal e do espaço velofaríngeo (Ko et al., 1999; Pegoraro-Krook et al., 2006; Birkent et al., 2009; Kim et al., 2013).

De acordo com Genaro, Yamashita e Trindade (2009) a avaliação perceptivo-auditiva é a principal maneira do fonoaudiólogo avaliar a nasalidade da fala e, de maneira indireta, investigar a FVF. Porém, por ser um método subjetivo e não haver uma padronização dos testes utilizados pode haver discordância intra e/ou interavaliadores. Por esse motivo, a experiência do avaliador pode causar influência (Lewis; Watterson; Houghton, 2002; Trindade; Yamashita; Gonçalves, 2007; Lee; Whitehill; Cioca, 2009; Brunnegắrd; Lohmander; Van Doorn, 2012; Scarmagnani et al., 2014). Portanto, é importante a utilização de métodos instrumentais para complementar a avaliação clínica, assim como para permitir um melhor planejamento e acompanhamento dos resultados de procedimentos 
terapêuticos (Trindade; Genaro; Dalston, 1997; Van Lierde; Wuyts; Bonte, 2007; Brunnegård; Lohmander; Van Doorn, 2012).

Diversas técnicas de avaliação e tipos de equipamentos, para identificar as alterações de nasalidade e do funcionamento do EVF, são empregadas na literatura. Dentre as técnicas, podem-se destacar o julgamento perceptivo-auditivo (Dalston; Vig, 1984; Haapenen et al., 1997; Harada et al., 2002; Lewis; Watterson; Houghton, 2002; Kummer; Briggs; Lee, 2003; Janulewicz et al., 2004; Chanchareonsook; Whitehill; Samman, 2007; Kummer et al., 2011; McComb et al., 2011; Brunnegård; Whitehill; Samman, 2012; Smedberg; Neovius; Lohmander, 2014) e a técnica fluxopressão (Dalston; Vig, 1984; Vallino, 1990; Trindade et al., 2003; Fukushiro; Trindade, 2005; Miguel; Genaro; Trindade, 2007; Fukushiro; Trindade, 2011) e dentre os equipamentos, o Nasômetro (Haapenen et al., 1997; Lewis; Watterson; Houghton, 2002; Soneghet et al., 2002; Trindade et al., 2003; Zemann et al., 2006; Chanchareonsook; Whitehill; Samman, 2007; Raimundo, 2007; Fukushiro; Trindade, 2011; Kim et al., 2013; Smedberg; Neovius; Lohmander, 2014; Blanton; Watterson; Lewis, 2015; Bunton, 2015).

Fletcher (1976) comparou os dados do Tonar II (instrumento precedente ao nasômetro) com o julgamento perceptivo-auditivo da nasalidade de 23 crianças, com fissura palatina reparada, e encontrou uma correlação razoável entre eles. Dalston e Warren (1986) compararam o Tonar II, a técnica fluxo-pressão e o julgamento perceptivo-auditivo para identificar hipernasalidade de 124 sujeitos (crianças e adultos, divididos em três grupos de acordo com o fechamento velofaríngeo) e encontraram um elevado grau de correlação entre as três técnicas utilizadas. Já Trindade et al. (2003) utilizaram a técnica fluxo-pressão e o nasômetro para avaliar os efeitos do avanço de maxila em 29 sujeitos, com fissura labiopalatina reparada, e apontaram a importância da avaliação instrumental. Lewis, Watterson e Houghton (2002) empregaram a técnica de julgamento perceptivo-auditivo e o nasômetro para analisar a relação entre seus achados e apontaram correlação de fraca a moderada. Já Brunnegård, Whitehill e Samman (2012) encontraram alta correlação entre o julgamento perceptivo-auditivo de juízes experientes, com os escores do nasômetro.

O nasômetro é um instrumento não invasivo que avalia de forma objetiva a nasalidade da fala e indiretamente, o funcionamento do EVF. Ou seja, oferece dados quantitativos sobre a nasalidade de fala, denominados escores de nasalância da fala 
(Fletcher, 1976). Devido ao alto índice de sensibilidade e especificidade para identificar hipernasalidade e hiponasalidade (Dalston; Neiman; González-Landa, 1993; Dalston et al., 1991), o nasômetro é bastante utilizado na literatura com diferentes propósitos e amostras populacionais (Seaver et al., 1991; Litzaw; Dalston, 1992; Anderson, 1996; Suguimoto; Pegoraro-Krook, 1996; Trindade; Genaro; Dalston, 1997; Scarsellone; Rochet; Wolfaardt, 1999; Nieminen et al., 2000; Di Ninno et al., 2001; Gildersleeve-Neumann et al., 2001; Soneghet et al., 2002; Sweeney; Sell; O'regan, 2004; Annelin; Hallongren, 2006; Pegoraro-Krook et al., 2006; Zemann et al., 2006; Narece, 2007; Mishima et al., 2008; Birkent et al., 2009; Brunnegárd; Van Door, 2009; Van Lierde et al., 2011; Okalidou et al., 2011; Abou-Elsaad et al., 2012; Brunnegård; Whitehill; Samman, 2012; Gerek et al., 2012; Ibrahim; Reilly; Kilpatrick, 2012; Bettens et al., 2013; Lee; Browne, 2013; lqueda et al., 2013; Karakoc et al., 2013; Kim et al., 2013; Thorp; Vimik; Stepp, 2013; El-Kassabi et al., 2014; Park et al., 2014; Tuzuner et al., 2014; Awan et al., 2015; Blanton; Watterson; Lewis, 2015; Bunton, 2015; D’haeseleer et al., 2015; Ha; Cho, 2015; Marino et al., 2015; Kim et al., 2016).

Diversos estudos em populações com línguas distintas foram realizados para padronizar os valores de nasalância e identificar fatores que podem influenciála (Seaver et al., 1991; Litzaw; Dalston, 1992; Anderson, 1996; Suguimoto; Pegoraro-Krook, 1996; Trindade; Genaro; Dalston, 1997; Scarsellone; Rochet; Wolfaardt, 1999; Di Ninno et al., 2001; Trindade et al., 2003; Sweeney; Sell; O'regan, 2004; Annelin; Hallongren, 2006; Narece, 2007; Mishima et al., 2008; Birkent et al., 2009; Brunnegắrd; Van Door, 2009; Okalidou et al., 2011; Gerek et al., 2012; Ibrahim; Reilly; Kilpatrick, 2012; Bettens et al., 2013; Karakoc et al., 2013; Lee; Browne, 2013; El-Kassabi et al., 2014; Park et al., 2014; Awan et al., 2015; D'haeseleer et al., 2015; Marino et al., 2015; Ha; Cho, 2015; Kim et al., 2016; Maturo 2017).

Suguimoto e Pegoraro-Krook (1996) padronizaram a porcentagem média de nasalância e o desvio padrão de 80 adultos falantes normais do português brasileiro $(12,6 \% \pm 3,4 \%$ para o texto oral e $43,5 \% \pm 5,2 \%$ para o texto nasal), e encontraram diferença entre os sexos dessa população, sendo a nasalância do sexo feminino maior que a do masculino para o texto nasal. Trindade, Genaro e Dalston (1997) também normalizaram os escores de nasalância de falantes normais do Português 
Brasileiro, característico da região de São Paulo, porém não houve diferença entre sexos em nenhum conjunto de frases. Por outro lado, a idade dos participantes apresentou diferenças significantes nos conjuntos de frases não nasais, apontando que quanto menor a idade, menor a nasalância. Maturo et al. (2017) avaliaram a influência do dialeto e do sexo nos valores de nasalância de adultos jovens falantes normais do Português, de dois estados brasileiros, e encontraram diferença entre os dialetos no texto oral, e, assim como Trindade, Genaro e Dalston (1997), não encontraram diferença entre os sexos.

Sweeney, Sell e O'regan (2004) avaliaram 70 crianças irlandesas (de quatro anos e 11 meses a 13 anos) com articulação, ressonância e voz normais, para normatização dos escores de nasalância durante a produção de uma amostra de fala padronizada. Não encontraram diferença significativa entre os sexos, mas sim entre os diferentes estímulos de fala. Raimundo (2007) também concluiu que os valores de nasalância variaram de acordo com as amostra de fala e que os valores médios obtidos das crianças com fissura labiopalatina e fala normal, foram semelhantes aos descritos na literatura em crianças normais falantes do português brasileiro. Okalidou et al. (2011) constataram que a língua grega não sofre influência do sexo dos indivíduos, porém tem menor nasalância que outras línguas já estudadas, como inglês, flamenco, espanhol e alemão.

Park et al. (2014) estabeleceram os escores normativos de nasalância para coreanos (108 crianças, entre 7 e 11 anos, e 108 adultos, entre 18 e 29 anos) com articulação, ressonância, voz e audição normais e descobriram que o sexo e a idade influenciam os escores de nasalância dessa população. Já Kim et al. (2016) também constataram a influência do sexo na nasalância e encontraram diferenças entre os quatro dialetos chineses estudados.

\subsection{Nasalância e cirurgia ortognática}

A cirurgia ortognática modifica as estruturas do sistema craniofacial responsáveis pelas funções da fala e provoca mudança no volume e na área da hipofaringe (Jakobsone; Stenvik; Espeland, 2011; Park et al., 2012), da orofaringe (Mattos et al., 2011; Park et al., 2012; Karabekmez; Kleinheinz; Jung, 2015) e da nasofaringe (Haapanen et al., 1997; Harada et al., 2002; Jakobsone; Stenvik; 
Espeland, 2011; Park et al., 2012; Karabekmez; Kleinheinz; Jung, 2015), cavidades importantes para ressonância.

Por esse motivo, os efeitos da cirurgia ortognática na articulação (Dalston; Vig, 1984; Bowers; Tobey; Shaye, 1985; Ruscello et al., 1986; Vallino, 1990; Ko et al., 1999; Ward et al., 2002; Janulewicz et al., 2004; Van Lierde et al., 2006; Hassan; Naini; Gill, 2007; O'Gara; Wilson, 2007), ressonância da fala e no funcionamento do EVF (Garber; Speidel; Marse, 1981; Dalston; Vig, 1984; Vallino, 1990; Haapanen et al., 1997; Ko et al., 1999; Harada et al., 2002; Trindade et al., 2003; Van Lierde et al., 2003; Janulewicz et al., 2004; Niemeyer et al., 2005; Van Lierde et al., 2006; Chanchareonssok; Samman; Whitehill, 2006; Chanchareonssok; Whitehill; Samman, 2007; O'Gara; Wilson, 2007; Chua et al., 2010; Pereira; Sell; Tuomainen, 2013) têm sido amplamente estudados.

Os estudos que investigaram as alterações de fala decorrentes da cirurgia ortognática não apresentaram um consenso nos resultados (Hassan; Naini; Gill, 2007). Alguns autores encontraram melhora automática da maioria ou de todos os erros articulatórios (Bowers; Tobey; Shaye, 1985; Ruscello et al., 1986; Vallino, 1990; Ko et al., 1999; Janulewicz et al., 2004; O'Gara; Wilson, 2007), enquanto outros alegam que a correção da deformidade dentofacial não é suficiente para corrigir essas alterações (Dalston; Vig, 1984; Ward et al., 2002; Van Lierde et al., 2006).

Ruscello et al. (1986) avaliaram as características de fala de 20 sujeitos com deformidades dentofaciais variadas no pré e no pós-operatório de cirurgia ortognática e observaram mudanças positivas na articulação após a correção cirúrgica. Vallino (1990) estudou, dentre outros aspetos, a articulação da fala de 34 sujeitos com deformidade dentofacial classe II e III (sem histórico de fissura lábio/palatina), antes e após a cirurgia ortognática e também observou melhora espontânea da articulação após a cirurgia. Já Van Lierde et al., (2006) realizaram um estudo com oito indivíduos com má oclusão classe II (sem histórico de fissura) visando determinar detalhadamente as características de ressonância, articulação e voz após osteotomia sagital com avanço mandibular e observaram que, após a cirurgia, a maioria dos pacientes mostrou um padrão articulatório idêntico (padrão normal ou perturbado) à condição pré-cirúrgica. Esses achados corroboram os de Dalton e Vig (1984), que avaliaram 40 mulheres adultas com deformidade 
dentofacial classe II e III (sem histórico de fissura), antes e após a cirurgia ortognática, para determinar os efeitos sobre a fala e, do mesmo modo, constataram que as alterações articulatórias existentes no pré-operatório se mantiveram após a cirurgia.

Relacionado às alterações na FVF e na nasalância da fala os estudos também apresentaram resultados inconsistentes ou contraditórios. À medida que alguns autores relataram que a cirurgia não traz prejuízos para fala ou nasalidade dos sujeitos (Garber; Speidel; Marse, 1981; Dalston; Vig, 1984; Vallino, 1990; Harada et al., 2002; Van Lierde et al., 2006; Kudo et al., 2014), outros apontaram que a osteotomia de avanço de maxila provoca mudança na área do EVF e, consequentemente, alteração na nasalidade e/ou nasalância (Haapanen et al., 1997; Ko et al., 1999; Ward et al., 2002; Trindade et al., 2003; Van Lierde et al., 2003; Janulewicz et al., 2004; Niemeyer et al., 2005; Chanchareonssok; Whitehill; Samman, 2007; O'Gara; Wilson, 2007; Chua et al., 2010; Pereira; Sell; Tuomainen, 2013).

Ward et al. (2002) avaliaram o impacto da má oclusão e da cirurgia ortognática nos padrões articulatórios e na nasalidade de cinco indivíduos com deformidades dentofaciais Classe II e III, antes e após a cirurgia ortognática de avanço de maxila, e os compararam com oito indivíduos controles. Os resultados mostraram padrões diferentes de alterações e apontaram aumento dos escores de nasalância, em 3 dos 5 casos, após a cirurgia.

Trindade et al. (2003) avaliaram a nasalância de 29 sujeitos, com fissura labiopalatina reparada, no pré e pós-operatório de avanço de maxila para avaliar os efeitos da cirurgia e constaram que, em alguns pacientes com fissura reparada a cirurgia ortognática, com avanço de maxila, modifica, em longo prazo (após 6 meses), a nasalância da fala. Janulewicz et al. (2004) confirmaram os achados de que pacientes com fissuras labiopalatinas, ou só de palato, estão predispostos à alteração da FVF após avanço da maxila, principalmente com a função borderline no pré-operatório. Igualmente, Pereira, Sell e Tuomainen (2013) concluíram que a osteotomia de maxila em indivíduos com fissuras, altera negativamente a FVF, provocando piora da hipernasalidade. Em contrapartida, esses achados não foram observados em indivíduos sem fissuras. 
Diante do exposto, pode-se notar que os efeitos da cirurgia ortognática em pacientes com deformidades dentofaciais não estão claros na literatura, pois apresentam variações de acordo com as deformidades, tipos cirúrgicos e populações estudas. 


\section{MATERIAIS E MÉTODOS}

\subsection{Considerações Éticas}

O trabalho, assim como o "Termo de consentimento livre e esclarecido", foi aprovado pelo Comitê de Ética em Pesquisa do Hospital das Clínicas da Faculdade de Medicina de Ribeirão Preto da Universidade de São Paulo, de acordo com o processo HCRP o 10870/2012 (ANEXO A). Todos os participantes concordaram e assinaram o referido termo e o estudo foi realizado baseado nos princípios éticos e legais que regem a pesquisa em seres humanos.

\subsection{Tipo de Estudo}

O presente estudo foi longitudinal, descritivo e observacional.

\subsection{Casuística}

Foram triados para este estudo 146 indivíduos. Destes, 91 voluntários, de ambos os sexos, com idade superior a 18 anos, alfabetizados e sem distinção de raça e nível socioeconômico, foram selecionados de acordo com critérios préestabelecidos. No decorrer do estudo 9 indivíduos foram excluídos (Figura 1).

\subsubsection{Caracterização da amostra}

\subsubsection{Grupo estudo}

Fizeram parte deste grupo 54 participantes, 23 indivíduos com deformidade dentofacial classe II (caracterizado por excesso de crescimento de maxila, associado ou não à retrognatia) com idade média de 29,4 anos $( \pm 6,3)$, sendo 18 do sexo feminino e 5 do sexo masculino; e 31 indivíduos com deformidade dentofacial classe III (caracterizado por deficiência maxilar associado ou não à prognatia) com média de idade de 27,2 anos $( \pm 8,1), 18$ do sexo feminino e 13 do sexo masculino (Tabela $1)$. 
Figura 1 - Fluxograma de distribuição da amostra

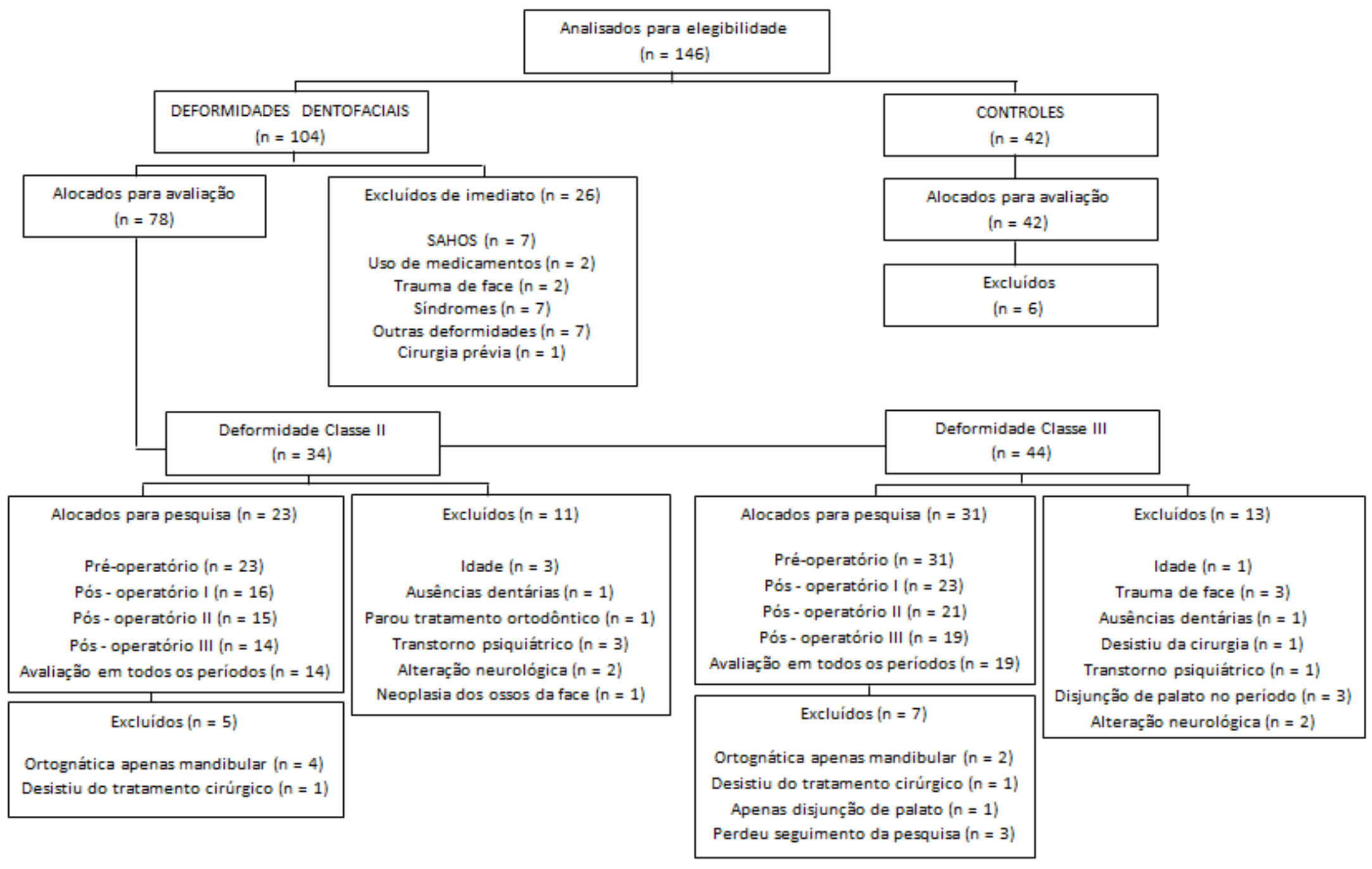

Fonte: Maturo (2017) 


\subsubsection{Grupo controle}

Compuseram este grupo 37 indivíduos, com média de idade de 25,3 anos ( \pm 6,5), 22 do sexo feminino e 15 do sexo masculino (Tabela 1).

Tabela 1 - Distribuição da amostra quanto aos grupos, número de sujeitos, sexo e idade, no período pré-operatório

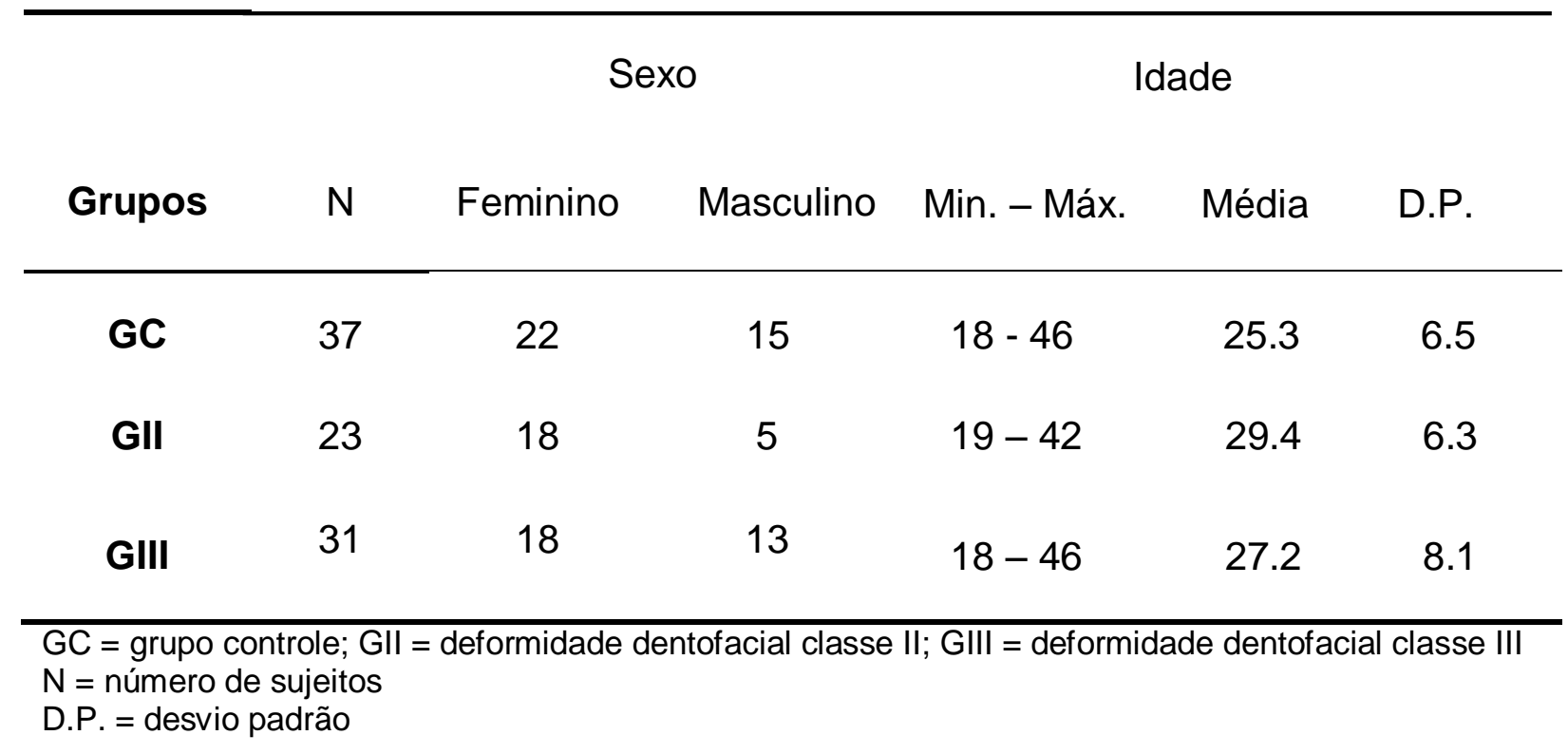

\subsubsection{Critérios de inclusão}

Grupo estudo: indivíduos com deformidade dentofacial classe II (caracterizado por excesso de crescimento de maxila) e classe III (caracterizado por deficiência maxilar) isoladas, com indicação de correção cirúrgica para tal deformidade e planejamento cirúrgico envolvendo a maxila, e com idade superior a 18 anos. Todos os participantes estavam em tratamento ortodôntico, antes e após a cirurgia, como procedimento habitual da rotina do Centro Integrado de Estudos das Deformidades da Face (CIEDEF).

Grupo controle: indivíduos sem deformidade dentofacial e com idade superior a 18 anos. 


\subsection{3 $\underline{\text { Critérios de exclusão }}$}

Grupo estudo: indivíduos com obstrução nasal evidente na avaliação, histórico de perda auditiva, alterações cognitivas e/ou neuromusculares; cirurgias ou traumas de face prévios ou com planejamento cirúrgico que não envolvesse a maxila. Tais dados foram investigados e confirmados por meio de entrevista, avaliação clínica e consulta ao prontuário médico.

Grupo controle: indivíduos com obstrução nasal crônica, histórico de perda auditiva, fissura labial e/ou de palato ou qualquer outra deformidade craniofacial, mesmo já reparada, com alterações de fala ou linguagem oral e com histórico de traumas ou cirurgias prévias em região cervicofacial.

Este estudo envolveu voluntários de várias regiões do país, como pode ser visto na Tabela 2.

Tabela 2 - Distribuição da amostra em porcentagem (\%), quanto aos grupos (GC, GII e GIII) e estado de origem

\begin{tabular}{cccc}
\hline Estados & GC & G II & G III \\
& $(\%)$ & $(\%)$ & (\%) \\
São Paulo & 59,46 & 86,96 & 83,9 \\
Minas Gerais & 40,54 & 13,04 & 6,5 \\
Rondônia & 0 & 0 & 3,2 \\
Paraná & 0 & 0 & 3,2 \\
Pará & 0 & 0 & 3,2 \\
\hline
\end{tabular}

\subsection{Procedimentos}

Após concordância em participar da pesquisa e assinado o Termo de Consentimento Livre e Esclarecido (APÊNDICE A e B), cada participante foi submetido individualmente a uma entrevista contendo dados de identificação, procedência e referente às alterações respiratórias, perda auditiva, realização de 
cirurgias orofaciais, traumas de face, alterações na alimentação e na voz (APÊNDICE C).

O grupo controle foi avaliado em apenas um período e o grupo estudo, em quatro períodos distintos: pré-operatório e pós-operatório I, II e III (Tabela 3).

Caso o participante apresentasse obstrução nasal, decorrente de fator externo (gripe, alergia), no dia da avaliação, o exame era reagendado para não causar influência no resultado da nasometria.

Os procedimentos foram realizados no Laboratório de Investigação do Sistema Estomatognático (LISE) e no Laboratório de Investigação de Voz e Fala (LIVF) da Faculdade de Medicina de Ribeirão Preto da Universidade de São Paulo. Durante as avaliações, os participantes permaneceram sentados em uma cadeira com encosto confortável e com os pés apoiados no chão.

Tabela 3 - Variação do período de avaliação, em dias

\section{Classe II}

Classe III

Períodos Variação (dias) Média (dias) Variação (dias) Média(dias)

\begin{tabular}{ccccc}
\hline Pré-operatório & 1 a 139 & 45 & 1 a 140 & 45 \\
P.O. I & 20 a 41 & 32 & 26 a 46 & 33 \\
P.O. II & 81 a 115 & 93 & 88 a 126 & 98 \\
P.O. III & 180 a 232 & 195 & 178 a 237 & 195 \\
\hline
\end{tabular}

P.O. = pós-operatório

\subsubsection{Avaliação clínica fonoaudiológica}

Foi avaliada a condição postural dos lábios, a posição da língua, a aparência do palato e a função de respiração de acordo com os itens do Protocolo de Avaliação Miofuncional Orofacial com escores (AMIOFE - de Felício; Medeiros; de Oliveira-Melchior, 2012) (ANEXO B). Avaliou-se também a morfologia da úvula e das tonsilas palatinas, a mobilidade do véu palatino, a ressonância e a inteligibilidade da fala (APÊNDICE D). 
Em seguida, os participantes foram orientados a realizar higiene nasal com água e soro fisiológico, para então ser avaliado o fluxo aéreo nasal utilizando o Espelho Nasal Milimetrado de Altmann - Pró-Fono ${ }^{\circledR}$ (Figura 2), e realizado o teste de emissão de ar nasal durante a produção dos fonemas /s/, /f/ e / /, a repetição de palavras e das provas de sopro e sopro com a língua projetada (APÊNDICE D). $O$ espelho foi higienizado e resfriado com algodão embebido em álcool $70 \%$ antes de cada avaliação, conforme instruções do fabricante.

Esta avaliação foi baseada na avaliação clínica descrita por Genaro, Yamashita e Trindade (2009), para indivíduos fissurados, que possuem alterações frequentes de ressonância.

\subsubsection{Nasometria}

Para a avaliação da nasalância foi utilizado o Nasômetro II $^{1}$, modelo 6400 (KayPENTAX, New Jersey - USA) (Figura 3), equipamento não invasivo que avalia de forma objetiva a nasalidade da fala. Ele é equipado com um capacete contendo dois microfones separados por uma placa horizontal, que deve ser posicionada entre o lábio superior e o nariz. Portanto, um microfone fica direcionado à cavidade nasal e o outro à cavidade oral, captando assim, sinais dos componentes nasais e orais da fala.

À medida que são lidos textos padronizados, apresentados na tela do computador, os sinais acústicos são filtrados, digitalizados e analisados por um software específico para nasometria. O cálculo da nasalância, realizado nas faixas de frequências de 300 e $600 \mathrm{~Hz}$, corresponde à razão numérica entre a energia acústica nasal e a energia acústica total (soma da energia nasal e oral) multiplicada por 100, pois a nasalância é expressa em porcentagem (\%). Portanto, teoricamente, esta pode variar de 0 (ausência de som emergindo pelo nariz) a 100\% (todo som emergindo pelo nariz).

O estímulo utilizado para o exame foi a leitura de 5 frases, do português brasileiro, destituídas de sons nasais (ZOO-BR ou texto oral) e 5 frases, do português brasileiro, destituídas de consoantes de pressão e contendo alta porcentagem de consoantes nasais (NASAL2-BR ou texto nasal), como proposto por Trindade, Genaro e Dalston (1997; ANEXO C). Foram considerados para a análise, 
os valores médios da nasalância da primeira emissão tecnicamente aceitável de cada palavra que constitui cada frase, ou seja, produzidas sem erros e dentro do limite de intensidade aceita pelo aparelho. Os valores de normalidade adotados foram, o limite máximo de $27 \%$ para o texto oral e o mínimo de $43 \%$ para o nasal (Trindade et al., 2003; Genaro; Yamashita; Trindade, 2009).

Antes de cada exame foi realizada a calibração do nasômetro, com fonte sonora do próprio aparelho, assim como sua higienização utilizando álcool $70 \%$.

Figura 2 - Espelho Milimetrado de Altmann - Pró-Fono ${ }^{\circledR}$

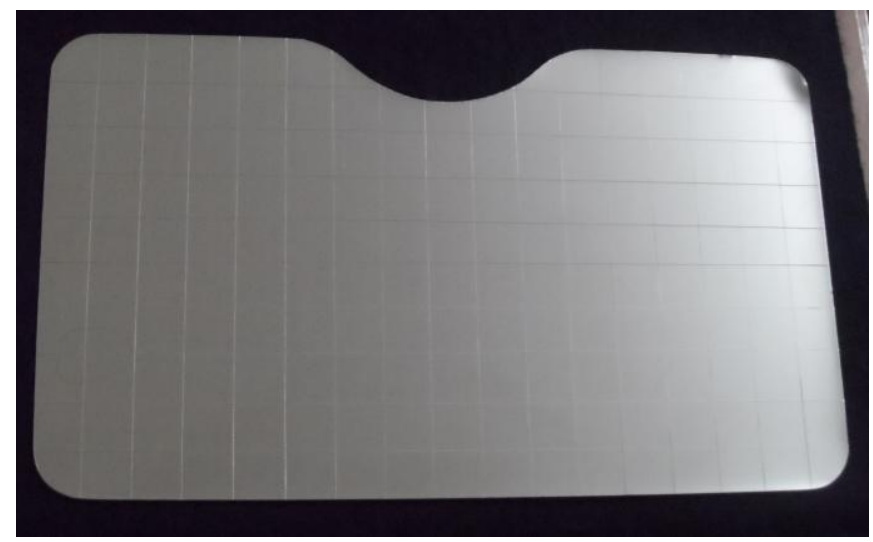

Fonte: Maturo (2017)

Figura 3 - Instrumento para mensuração da nasalância
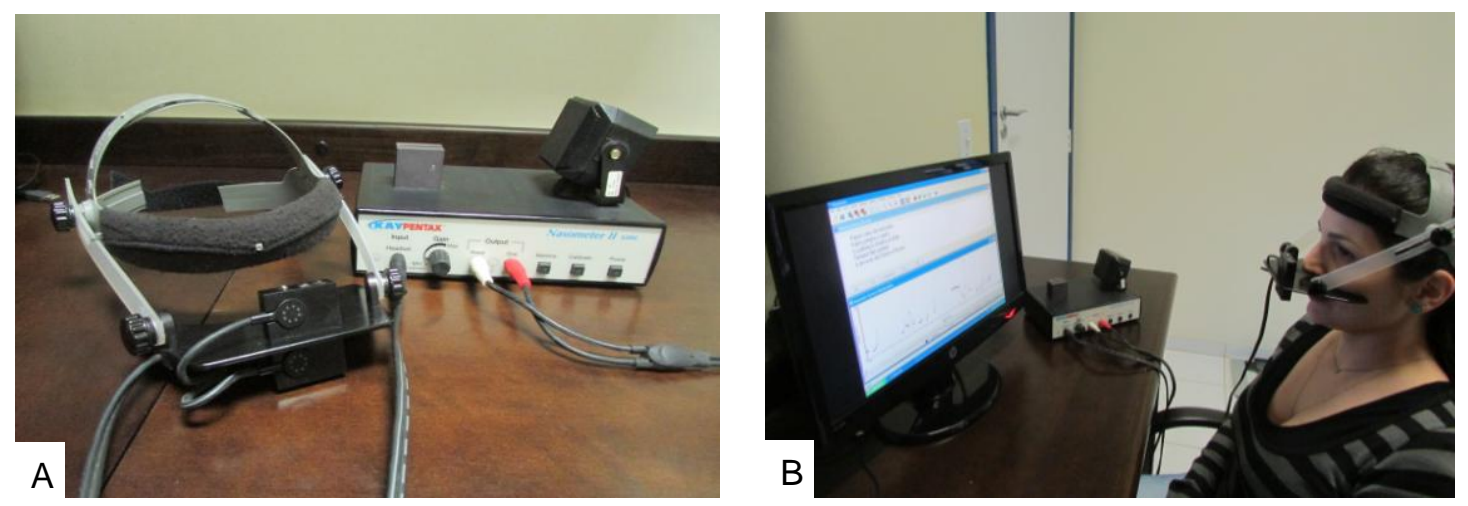

A. Nasômetro II mod. 6400, KayPENTAX ${ }^{\circledR}$; B. Exame de nasometria. Fonte: Maturo (2017)

\subsubsection{Tratamentos realizados}

Os participantes foram submetidos ao tratamento de cirurgia ortognática de acordo com sua deformidade e conforme programação da equipe do CIEDEF (Tabela 4). 
Tabela 4 - Distribuição da amostra quanto aos grupos (Gll e GIII) e procedimentos/técnicas cirúrgicas

Técnica cirúrgica

G II G III

Le fort I (avanço)

Le fort I (avanço e intrusão)

02

Le fort I + mentoplastia

01

Le fort I (avanço) + sagital de mandíbula

32

Le fort I (avanço e intrusão) + sagital de mandíbula

$4 \quad 1$

Le fort I (avanço) + sagital de mandíbula + mentoplastia

40

Le fort I (intrusão) + sagital de mandíbula + mentoplastia

20

Le fort I (avanço e intrusão) + sagital de mandíbula + mentoplastia

10

Le fort I + sagital de mandíbula + mentoplastia + septoplastia 10

Le fort I + sagital de mandíbula + septoplastia 10

Le fort I (avanço) + vertical de mandíbula

03

Le fort I (avanço e intrusão) + vertical de mandíbula

Le fort I + vertical de mandíbula + septoplastia

$\begin{array}{ll}0 & 1 \\ 0 & 1\end{array}$

Total

$16 \quad 23$

GII = grupo deformidade dentofacial classe II; GIII = grupo deformidade dentofacial classe III

Todos os participantes foram acompanhados e realizaram atendimento fonoaudiológico (terapia miofuncional orofacial), como procedimento padrão do CIEDEF, mas nenhum teve como enfoque a terapia de ressonância da fala (Tabela 5). 
Tabela 5 - Porcentagem de sujeitos que realizaram fonoterapia de acordo com os grupos (Gll e GIII) e procedimentos fonoterápicos, no período pósoperatório

\begin{tabular}{lcc}
\hline \multicolumn{1}{c}{ Exercícios } & $\begin{array}{c}\text { G II } \\
\text { (\%) }\end{array}$ & $\begin{array}{c}\text { G III } \\
\text { (\%) }\end{array}$ \\
\hline Termoterapia / Massagem / Orientação sobre alimentação & 100 & 100 \\
Movimentos mandibulares espontâneos & 100 & 100 \\
Movimentos mandibulares com tração & 18,8 & 18,2 \\
Exercícios para língua & 43,8 & 59,1 \\
Exercícios para lábios & 31,3 & 9,1 \\
Estimulação da sensibilidade orofacial & 75 & 50 \\
Treino da mastigação & 43,8 & 54,6 \\
Treino da deglutição & 12,5 & 27,3 \\
\hline Gll = grupo deformidade dentofacial classe II; GIII = grupo deformidade dentofacial classe III
\end{tabular}

GII = grupo deformidade dentofacial classe II; GIII = grupo deformidade dentofacial classe III

\subsection{Análises estatísticas}

Foi realizado o teste estatístico de Análise de Variância (ANOVA) - two-way para analisar os fatores grupo e sexo e sua interação.

Para verificar a distribuição dos sexos nos grupos controle (GC), deformidade dentofacial classe II (GII) e classe III (GIII), foi utilizado o teste Quiquadrado.

Para analisar os escores de nasalância nos quatro períodos de avaliação, foram considerados apenas os indivíduos dos grupos Gll e GIII que tinham avaliação nasométrica em todos os períodos. Para essa análise foi realizado o teste estatístico ANOVA two-way, para medidas repetidas, e o pós-teste de Tukey HSD.

O software utilizado para as análises foi o JMP - Pró 10.0 (SAS - Institute; Cary - North Carolina,USA) e foram consideradas diferenças significantes de $p<0,05$. 
5. RESULTADOS 


\section{RESULTADOS}

Os valores médios e os desvios-padrão dos escores de nasalância dos indivíduos controles e com deformidades dentofaciais classe II e III, estão listados na Tabela 6. Como pode ser observado, no período pré-operatório, os grupos apresentaram médias dentro dos valores de normalidade, de acordo com os valores de referência (máximo de $27 \%$ para o texto oral e mínimo de $43 \%$ para o nasal).

Tabela 6 - Valores médios e desvio padrão de nasalância (\%) dos grupos Controle, Classe II e Classe III no pré-operatório

\begin{tabular}{cccc} 
& & Nasalância (\%) \\
Texto & $\mathrm{GC}$ & $\mathrm{G}$ II & $\mathrm{N}=23$ \\
Nasal & $48.1(5.9)$ & GIII & $\mathrm{N}=31$ \\
Oral & $12.0(5.3)$ & $14.9(7.0)$ & $45.4(9.7)$ \\
\hline
\end{tabular}

$\mathrm{GC}=$ grupo controle; GII = deformidade dentofacial classe II; GIII = deformidade dentofacial classe III $\mathrm{N}=$ números de sujeitos

Para análise dos fatores grupo e sexo, no texto nasal, não houve interação $(p=0,50)$ nem diferenças entre os valores de nasalância para esses fatores analisados $(p=0,22$ e $p=0,93)$. Da mesma forma, não houve interação entre os fatores grupo e sexo para o texto oral $(p=0,89)$, porém, o fator sexo apresentou diferença significativa nos valores de nasalância $(p=0,04)$. O teste $t$ de Student apontou valores maiores no sexo feminino $(p=0,041)$. Esses dados podem ser verificados na Tabela 7 . 
Tabela 7 - Influência e interação dos fatores grupo e sexo nos valores médios de nasalância para os textos nasal e oral

Texto Nasal Texto oral

\begin{tabular}{ccccc} 
Fatores & Razão F & p-valor & Razão F & p-valor \\
\hline Grupo & 1,55 & 0,22 & 2,02 & 0,14 \\
Sexo & 0,01 & 0,93 & 4,35 & $0,04^{* \#}$ \\
Grupo*Sexo & 0,70 & 0,50 & 0,11 & 0,90
\end{tabular}

ANOVA two-way - *Valores estatisticamente significantes $(\mathrm{p}<0,05)$; \#Teste t de Student - Feminino $>$ masculino

Devido à influência apresentada pelo fator sexo foi aplicado o teste Quiquadrado, para analisar a distribuição dos sexos nos grupos. O teste mostrou proporção semelhante de homens e mulheres nos três grupos (Tabela 8), por esse motivo, os sexos foram agrupados para as demais análises.

Tabela 8 - Relação da proporção dos sexos nos grupos

\begin{tabular}{ccccc}
\hline Grupos & Feminino (\%) & Masculino (\%) & & \\
\hline GC & 59,46 & 40,54 & Qui-quadrado & p-valor \\
GII & 78,26 & 21,74 & 2,824 & 0.2437 \\
GIII & 58,06 & 41,94 & & \\
\hline
\end{tabular}

Teste Qui-quadrado - valores significantes $p<0,05$.

$\mathrm{GC}=$ controle; $\mathrm{GII}$ = deformidade dentofacial classe II; GIII = deformidade dentofacial classe III

Para análise de variância dos períodos de avaliação foram incluídos somente os indivíduos que possuíam a avaliação nasométrica nos quatro períodos (préoperatório e pós-operatórios I, II e III). Sendo assim, 14 indivíduos compuseram o grupo Gll e 19, o GIII.

A tabela 9 demonstra que os fatores grupo e tempo, não mostraram interação nos textos nasal $(p=0,12)$ e oral $(p=0,15)$. $O$ fator grupo não mostrou influência sobre os valores de nasalância para ambos os textos (nasal $p=0,81$ e oral $p=0,14$ ), já 0 fator tempo, mostrou diferença estatística para o texto oral $(p=0,02)$. 
Tabela 9 - Influência e interação dos fatores grupo e tempo nos valores médios de nasalância para os textos nasal e oral

\begin{tabular}{ccccc}
\hline Fatores & Razão $F$ & $\begin{array}{c}\text { Texto Nasal } \\
\text { p-valor }\end{array}$ & Razão F & p-valor \\
\hline Grupo & 0,06 & 0,81 & 0,52 & 0,48 \\
Tempo & 1,85 & 0,14 & 3,49 & $0,02^{*}$ \\
Grupo*Tempo & 1,55 & 0,21 & 1,80 & 0,15 \\
\hline
\end{tabular}

ANOVA two-way para medidas repetidas - *Valores significantes $(p<0.05)$

Foi constatada diferença significante no texto oral, entre os períodos préoperatório e pós-operatório III, por meio do teste t de Student (Tabela 10).

Tabela 10 - Valores médios e desvios padrão de nasalância (\%) dos grupos Classe II e Classe III, nos diferentes períodos de avaliação nasométrica

\section{Nasalância (\%)}

Pré-op P.O. I P.O. II P.O. III p-valor Pré-op P.O. I P.O. II P.O. III p-valor
GII $(\mathrm{N}=14)$
$48(6) \quad 46(7) \quad 46(7) \quad 50(9)$
$14(8)^{\mathrm{A}} 13(6)^{\mathrm{AB}} 14(9)^{\mathrm{AB}} 17(11)^{\mathrm{B}}$
0.14
$0.01^{*}$
GIII ( $N=19)$
44 (9) 48 (12) 47 (8) 48 (10)
$11(5)^{\mathrm{A}} 16(8)^{\mathrm{AB}} 13(7)^{\mathrm{AB}} 14(8)^{\mathrm{B}}$

\footnotetext{
${ }^{*}$ ANOVA two-way para medidas repetidas; post-test Tukey HSD - Valores significantes $(p<0.05)$. Médias seguidas de letras diferentes diferem estatisticamente.

GII = deformidade dentofacial classe II; GIII = deformidade dentofacial classe III

Pré-op. = pré-operatório; P.O.I = pós-operatório aproximado de 1 mês; P.O.II = pós-operatório aproximado de 3 meses; P.O.III = pós-operatório aproximado de 6 meses.

$\mathrm{N}=$ números de sujeitos
} 


\section{DISCUSSÃO}

A nasalância, correlato acústico da nasalidade, é uma grandeza física que corresponde à quantidade relativa de energia acústica emitida pela cavidade nasal durante a fala (Fletcher, 1976). Inúmeros fatores podem influenciá-la, como por exemplo, sexo do indivíduo, tamanho e formato das cavidades nasal e oral, deformidades dentofaciais e cirurgias bucomaxilares.

As deformidades dentofaciais são caracterizadas por alterações na relação maxilomandibular e podem trazer como consequência, a modificação no tamanho e configuração da cavidade oronasal. Sendo assim, indivíduos com alterações dentoesqueléticas estão mais propensos a terem alterações de nasalidade.

No presente estudo não foram encontradas diferenças significativas nos valores de nasalância entre os grupos GC, GIl e GIII, no período pré-operatório. Os valores médios apresentados estão dentro dos padrões de normalidade, mostrando que, de um modo geral, as alterações anteroposteriores da relação maxilomandibular e consequentemente do espaço aéreo faríngeo, não afetaram a nasalidade de fala desses indivíduos. Talvez a dimensão da deformidade dentofacial possa influenciar nos valores de ressonância, ou seja, não basta ter a deformidade, mas deve-se considerar sua gravidade.

Alguns autores relatam que entre as deformidades dentofaciais há uma variação das dimensões faríngeas das vias aéreas superiores. Kirjavainen e Kirjavainen (2007) avaliaram as estruturas aéreas superiores de 40 crianças saudáveis com má oclusão classe II, divisão 1, tratadas com aparelho ortopédico extrabucal (com apoio na cervical) e compararam com a cefalometria de 80 crianças controles, com relação molar classe I. Concluíram que, antes do tratamento, as crianças com a má oclusão classe II divisão 1, mesmo sem retrognatia, apresentaram a nasofaringe igual ou até maior que os controles, porém, a oro e hipofaringe eram mais estreitas.

Grauer et al. (2009) analisaram o volume e formato da via aérea faríngea, por meio de tomografia computadorizada cone-beam, fotografia facial e radiografia cefalométrica lateral, de 62 pacientes com deformidades dentofaciais (21 classe I, 21 classe II e 21 classe III, entre 17-46 anos) e também encontraram volume menor nos pacientes classe II esquelética, além de diferença na orientação e formato das vias 
aéreas entre classe II e classe III. Kim Y-J et al. (2010) e El e Palamo (2011) igualmente observaram que em pacientes com retrognatia o volume total das vias aéreas foi menor, comparado a sujeitos sem deformidades.

Alves Jr et al. (2012) evidenciaram que as dimensões do espaço aéreo faríngeo em crianças com relação de crescimento maxilomandibular balanceada é maior que em crianças com deficiência de crescimento anteroposterior de mandíbula. Já Hong et al. (2011) examinaram 31 adultos com má-oclusão classe III esquelética, compararam com 29 adultos com oclusão classe I e concluíram que o volume superior do espaço faríngeo é maior em pacientes com má-oclusão classe III esquelética, e que esse aumento de volume está correlacionado com medidas que caracterizam a posição anteriorizada da mandíbula. Martin, Muelas e Viñas (2011) também compararam pacientes, caucasianos saudáveis, com má-oclusão classe III $(\mathrm{N}=71)$ com pacientes com oclusão ideal $(\mathrm{N}=90)$, e uma das conclusões foi que a dimensão da faringe é maior em pacientes classe III.

Em outro estudo realizado por El e Palamo (2013), os autores confirmaram que sujeitos com deformidade dentofacial classe II, por retrognatia de mandíbula, apresentam menor volume das vias aéreas naso e orofaríngea, principalmente, quando comparados com sujeitos com deformidade dentofacial classe III, devido à prognatia. Castro-Silva et al. (2015) avaliaram o espaço aéreo faríngeo de 60 pacientes adultos saudáveis, com deformidade dentofacial classes I, II e III, por meio de tomografia computadorizada cone-beam, avaliação clínica e cefalométrica. Os resultados mostraram concordância que a média do volume e área do espaço aéreo faríngeo, em pacientes classe III, foi estatisticamente maior, comparado a pacientes classe I e II. Houve também uma diferença significativa para os valores de volume entre os pacientes classes I e II, sendo maior para os classe I.

Apesar do volume e da área do espaço aéreo faríngeo serem influenciados pelos diferentes padrões das más oclusões esqueléticas (Kirjavainen; Kirjavainen, 2007; Grauer et al., 2009; Kim Y-J et al., 2010; Martin; Muelas; Viñas, 2011; Hong et al., 2011; Alves Jr et al., 2012; El; Palamo, 2011, 2013; Castro-Silva et al., 2015) e poderem influenciar as características da nasalidade, isto não foi observado neste estudo.

A grande maioria dos sujeitos, nos três grupos, apresentou respiração nasal no período pré-operatório. Alves et al. (2008) compararam as dimensões do espaço 
aéreo faríngeo de 60 sujeitos respiradores nasais, 30 classe II e 30 classe III, e concluíram que o espaço aéreo superior desses pacientes não diferiram estatisticamente entre os padrões esqueléticos classe II e III. Esse fato pode justificar não ter havido diferença dos escores de nasalância entre os grupos GC, GII e GIII. Pode-se supor ainda que há uma adaptação no mecanismo de funcionamento das estruturas velofaríngeas, em conformidade com as alterações dentoesqueléticas anteroposteriores, não causando prejuízo na sua função.

Analisando os dados do texto oral na Tabela 7, o sexo foi apontado como um fator de influência da nasalância, indicando valores mais altos nas mulheres, porém, a proporção dos sexos verificada nos três grupos foi semelhante (Tabela 8).

Valores maiores de nasalância do sexo feminino são evidenciados por vários estudos na literatura (Suguimoto; Pegoraro-Krook, 1996; Rochet et al, 1998; Di Ninno et al., 2001; Van Lierde et al., 2001; Mishima et al., 2008; Karakoc et al., 2013; Park et al., 2014; Kim et al., 2016). Suguimoto e Pegoraro-Krook (1996) avaliaram a nasalância de 80 adultos falantes normais do português brasileiro, pela leitura de textos nasais e textos orais, e observaram que a nasalância do sexo feminino foi maior que a do masculino, para o texto nasal. Van Lierde et al.(2001) pesquisaram o valor de nasalidade de 58 jovens adultos falantes normais do holandês e os resultados apontaram, igualmente, as mulheres como tendo maior nasalância que os homens, no conjunto nasal.

Di Ninno et al. (2001) avaliaram a nasalância de 180 indivíduos falantes normais do português brasileiros, sem deformidade dentofacial ou outras anomalias, e também observaram que os valores de nasalância do sexo feminino foram maiores. Mishima et al. (2008) encontraram valores maiores nas mulheres em todos os estímulos testados na língua japonesa, assim como Kim et al. (2016), que também encontraram diferenças em todos os estímulos testados no mandarim, sendo os valores maiores de nasalância para sexo feminino.

Apesar das mulheres apresentarem valores mais altos de nasalância, o sexo não foi considerado fator influenciador neste estudo, pois tal fator mostrou-se igualmente presente nos 3 grupos.

O fato das mulheres apresentarem valores mais altos de nasalância pode ser atribuído às diferenças no mecanismo do EVF e no comprimento do trato vocal, existentes entre homens e mulheres (Van Lierde et al., 2001; Van Lierde et al., 2003; 
Mishima et al., 2008; Okalidou et al., 2011; Abou-Elsaad et al., 2012; Kummer, 2013). Já a diferença encontrada entre os estímulos, oral no presente estudo e nasal nos de Suguimoto e Pegoraro-Krook (1996) e de Van Lierde et al. (2001), pode ser atribuída às variações das dimensões das vias aéreas superiores encontradas nas deformidades dentofaciais.

Dos 54 indivíduos participantes com deformidades dentofaciais, 33 receberam intervenção cirúrgica durante o período do estudo, sendo submetidos ao avanço de maxila, pela técnica Le Fort I, associado ou não a osteotomia de mandíbula e/ou mento, e por técnicas cirúrgicas adequadas de acordo com o planejamento prévio.

Os efeitos da cirurgia ortognática, com avanço de maxila (Le fort I) e/ou movimentação da mandíbula, mostrou-se presente nos escores de nasalância do texto oral. A média dos valores no período P.O. III apresentou aumento significante, porém, ainda se manteve dentro dos padrões de normalidade. Kudo et al. (2014) encontraram resultados semelhantes e atribuíram o ocorrido ao fato de pacientes com deformidades dentofaciais, sem fissuras de lábio e/ou palato, possuírem um alto grau de adaptação da região faríngea após o avanço de maxila. Portanto, a cirurgia teve efeito significativo na nasalância da fala, contudo, não alterou as características da nasalidade dos indivíduos avaliados. Esses achados corroboram os de Dalston e Vig (1984), Zemann et al. (2006), Pereira, Sell e Tuomainen (2013) e Kudo et al. (2014).

De acordo com a literatura o avanço de maxila modifica o espaço nasofaríngeo, envolvido na ressonância da fala, e as cirurgias na mandíbula afetam, principalmente, o espaço hipofaríngeo. Mattos et al. (2011), após revisão de literatura, afirmaram que a cavidade orofaríngea diminui significativamente com o recuo da mandíbula e discretamente, com a cirurgia bimaxilar pra correção da classe III esquelética, enquanto aumenta com a cirurgia de avanço maxilomandibular.

Alguns autores ainda relatam que o avanço de maxila traz prejuízo para a FVF (Haapanen et al., 1997; Ko et al., 1999; Ward et al., 2002; Trindade et al., 2003; Van Lierde et al., 2003; Janulewicz et al., 2004; Niemeyer et al., 2005; Chanchareonssok; Whitehill; Samman, 2007; O'Gara; Wilson, 2007; Chua et al., 2010; Pereira; Sell; Tuomainen, 2013) e consequentemente, alteração de ressonância. De acordo com llg (1999), nos casos de avanço de maxila, existe um 
deslocamento anterior do palato mole, o que pode prejudicar o selamento posterior do véu palatino, causando a incompetência velofaríngea.

Em pacientes com fissura labiopalatina reparada a deterioração da FVF é bem evidenciada. Haapanen et al. (1997) relatam que o avanço de maxila causa um pequeno, mas significativo, prejuízo no FVF e piora os escores de nasalância no pós-operatório de pacientes com fissura labiopalatina prévia. Ko et al. (1999) afirmaram que o efeito do avanço de maxila na fala pode ter benefícios na melhoria da articulação, mas compromete o fechamento velofaríngeo, pois aumenta a distância da nasofaringe. Em outro estudo, feito pelos mesmos autores no mesmo ano, envolvendo sujeitos com vários tipos de fissuras e submetidos ao avanço de maxila por distração osteogênica, eles concluiram que o aumento da profundidade da nasofaringe pode comprometer o fechamento velofaríngeo, piorando a hipernasalidade.

Ward et al. (2002) avaliaram cinco indivíduos com deformidades dentofaciais classes II e III, antes e após a cirurgia ortognática de avanço de maxila, e os compararam com oito indivíduos controles. Os resultados apontaram piora da nasalidade após a cirurgia. Trindade et al. (2003) avaliaram a nasalância de 29 sujeitos com fissura labiopalatina reparada, no pré e pós-operatório de avanço de maxila, e constaram que, nesses pacientes, o avanço de maxila aumenta a área velofaríngea e piora, em longo prazo, a nasalância. Confirmando esses achados, Janulewicz et al. (2004) evidenciaram que pacientes com fissuras labiopalatinas ou só de palato, são predispostos à alteração da FVF após avanço da maxila, principalmente, com a função borderline no pré-operatório.

Niemeyer et al. (2005) verificaram as modificações da ressonância da fala, após avanço de maxila, em 42 sujeitos (24 homens e 18 mulheres) com fissura labiopalatina reparada e com presença de deformidade dentofacial, caracterizada por mordida cruzada anterior associada ou não com mordida cruzada posterior. Com os resultados, sugerem que a cirurgia ortognática em sujeitos com fissura palatina pode interferir na ressonância, causando ou aumentando o grau de hipernasalidade. Igualmente, O'Gara e Wilson (2007) relataram que sujeitos com fissuras lábio/palatinas ou com FVF borderline, podem ter uma deterioração da FVF após cirurgia de maxila. 
A literatura aponta também efeitos diferentes da cirurgia ortognática comparando pacientes com e sem fissuras labiopalatinas, como observado nos estudos de Pereira, Sell e Tuomainen (2013) e Kudo et al. (2014). Pereira, Sell e Tuomainen (2013) avaliaram os efeitos do avanço de maxila em sujeitos com e sem fissura labiopalatina e concluíram que, em pacientes fissurados há um impacto negativo na ressonância nasal e no funcionamento do EVF, já em sujeitos não fissurados o avanço não causou efeito sobre a ressonância ou o funcionamento do EVF. Kudo et al (2014) avaliaram as mudanças na fala e no espaço faríngeo após avanço de maxila em pacientes com e sem fissura de palato. Observaram que a nasalância dos sujeitos com fissura teve uma piora temporária após a cirurgia, enquanto que a nasalância dos sujeitos não fissurados, não sofreu alteração.

Em outros estudos com pacientes com deformidade dentofacial e sem fissuras de lábio e/ou palato resultados semelhantes foram encontrados. Dalston e Vig (1984) investigaram os efeitos da cirurgia ortognática em 40 mulheres adultas e observaram que não houve diferença na FVF, tanto no pré como no pós-operatório. Mas observaram que a diminuição da resistência nasal e o equilíbrio da ressonância oronasal, devido ao reposicionamento da maxila, mostrou correlação, porém, essa mudança não foi notada como alteração de nasalidade (hipo ou hipernasal).

Van Lierde et al. (2006) relatam que a cirurgia de avanço mandibular pela osteotomia sagital bilateral do ramo da mandíbula não teve impacto nas características da nasalidade, nem nos valores de nasalância, e atribuíram os resultados à competência da válvula velofaríngea desses sujeitos no pré-operatório.

Zemann et al. (2006) analisaram os efeitos da cirurgia bimaxilar em 20 indivíduos, não fissurados e com fala normal, e concluíram que todos os sujeitos mostraram mudanças nos valores de nasalância no pós-operatório de 6 semanas, porém, os valores obtidos não tiveram impacto significante nas características da nasalidade da fala.

A diferença na nasalância, evidenciada, este estudo, apenas no texto oral, pode ser relacionada ao aumento da cavidade de ressonância oronasal após o avanço de maxila. Pegoraro-Krook et al. (2006), Birkent et al. (2009) e Kim et al (2013) relatam que o volume e o tamanho da cavidade nasal podem influenciar os valores de nasalância, sendo assim, justifica os resultados encontrados neste estudo. 
Para o presente estudo, foram incluídos apenas pacientes com algum grau de alteração em maxila e que foram submetidos, obrigatoriamente, à cirurgia de maxila, combinada ou não à osteotomias de mandíbula.

A maioria dos pacientes, no pré-operatório, apresentava FVF adequada, de acordo com os valores nasométricos, ocasionando a manutenção da classificação da nasalidade. Todavia, numa análise individual, os poucos pacientes que apresentavam hipernasalidade prévia mostraram piora dos escores na avaliação final do pós-operatório e, os que apresentavam hiponasalidade tiveram uma discreta melhora ou mantiveram os escores. Talvez, se para a análise dos dados, os indivíduos tivessem sido separados de acordo com a classificação da nasalidade e/ou o tipo de cirurgia realizada, os resultados poderiam ser diferentes. Entretanto, devido a grande variedade de procedimentos cirúrgicos realizados além do avanço de maxila, a amostra de sujeitos em cada grupo seria pequena, tornando a análise dos resultados pouco confiável.

Dessa maneira, sugerem-se novos estudos, com uma amostra maior de indivíduos, para avaliar e compreender os efeitos dos diferentes procedimentos cirúrgicos na nasalância da fala. Contudo, os resultados do presente estudo levam um alerta para a equipe de cirurgia ortognática, para que conste, no planejamento ou preparo cirúrgico, a investigação da ressonância da fala, mesmo que o caso não evidencie uma insuficiência ou disfunção velofaríngea. Justifica-se assim, a importância da presença de um fonoaudiólogo na equipe, para que atue em avaliações e nas discussões conjuntas do planejamento dos casos. 
7. CONCLUSÕES 


\section{CONCLUSÕES}

A média dos valores de nasalância, tanto dos indivíduos com deformidade dentofacial classe II quanto dos indivíduos classe III, está dentro dos padrões de normalidade estabelecidos para a pesquisa (máximo de $27 \%$ para o texto oral e mínimo de $43 \%$ para o nasal), portanto:

- O tipo da deformidade dentofacial parece não afetar a nasalância dos indivíduos.

No pós-operatório as médias dos valores de nasalância se mantiveram dentro dos padrões de normalidade, porém, houve um aumento significativo nos escores do texto oral, ou seja:

- A cirurgia ortognática provocou efeito na nasalância de fala, no período de 6 meses, para o texto oral, porém, não provocou alteração nas características da nasalidade de fala. 


\section{REFERÊNCIAS BIBLIOGRÁFICAS ${ }^{2}$}

Abou-Elsaad T, Quriba A, Baz H, Elkassaby R. Standardization of Nasometry for Normal Egyptian Arabic Speakers. Folia Phoniatr Logop. 2012; 64(6):271-7.

Al-Moraissi EA, Al-Magaleh SM, Iskandar RA, Al-Hendi EA. Impact on the pharyngeal airway space of different orthognathic procedures for the prognathic mandible. Int J Oral Maxillofac Surg. 2015 Sep;44(9):1110-8.

Alves PVM, Zhao L, O'Gara M, Patel PK, Bolognese AM. Three-dimensional cephalometric study of upper airway space in skeletal class II and III healthy patients. J Craniofac Surg. 2008 Nov;19(6):1497-507.

Alves M Jr, Franzotti ES, Baratieri C, Nunes LK, Nojima LI, Ruellas AC. Evaluation of pharyngeal airway space amongst different skeletal patterns. Int $\mathrm{J}$ Oral Maxillofac Surg. 2012 Jul;41(7):814-9.

Anderson RT. Nasometric values for normal Spanish-speaking females: a preliminary report. Cleft Palate Craniofac J. 1996 Jul;33(4):333-6.

Annelin M, Hallongren M. Influence of city, age and gender on nasalance scores for two Swedish populations [dissertation]. Höstterminen: Umeå Universitet, Institutionen för klinisk vetenskap, 2006.

Araujo A. Cirurgia Ortognática. São Paulo: Santos; 1999. 374 p.

Awan SN, Bressmann T, Poburka B, Roy N, Sharp H, Watts C. Dialectical effects on nasalance: a multicenter, cross-continental study. J Speech Lang Hear Res. 2015 Feb;58(1):69-77.

Baken RJ. Clinical measurement of speech and voice. Boston: College-Hill Press; 1997. 518p.

Behlau M, Madazio G, Feijó D, Pontes P. Avaliação de voz. In: Behlau M. Voz: o livro do especialista. Rio de Janeiro: Revinter; 2001. p. 85-176.

Berretin-Felix G, Jorge TM, Genaro KF. Intervenção fonoaudiológica em pacientes submetidos a cirurgia ortognática. In: Fernandes FDM, Mendes BCA, Navas ALPGP, organizadores. Tratado de Fonoaudiologia. 2a ed. São Paulo: Roca; 2009. Capítulo 58, p. 545-57.

Bettens K, Wuyts FL, De Graef C, Verhegge L, Van Lierde KM. Effects of age and gender in normal-speaking children on the nasality severity index: an objective multiparametric approach to hypernasality. Folia Phoniatr Logop. 2013;65(4):185-92.

Birkent H, Erol U, Ciyiltepe M, Eadie TL, Durmaz A, Tosun F. Relationship between nasal cavity volume changes and nasalance. J Laryngol Otol. 2009 Apr;123(4):40711.

\footnotetext{
${ }^{2}$ De acordo com: UNIVERSIDADE DE SÃO PAULO. Sistema Integrado de Bibliotecas. Diretrizes para apresentação de dissertações e teses da USP: documento eletrônico e impresso Parte IV (Vancouver) $3^{\text {a }}$ ed. São Paulo: SIBi/USP, 2016
} 
Blanton A, Watterson $\mathrm{T}$, Lewis $\mathrm{K}$. The differential influence of vowels and palatal covering on nasalance scores. Cleft Palate Craniofac J. 2015 Jan;52(1):82-7

Bowers J, Tobey EA, Shaye R. An acoustic-speech study of patients who received orthognathic surgery. Am J Orthod. 1985 Nov;88(5):373-9.

Brunnegård $\mathrm{K}$, Lohmander $\mathrm{A}$, Van Doorn J. Comparison between perceptual assessments of nasality and nasalance scores. Int. J. Lang. Commun. Disord. 2012 Set-Oct; 47(5):556-66.

Brunnegård K, Van Doorn J. Normative data on nasalance scores for Swedish as measured on the Nasometer: influence of dialect, gender, and age. Clin. Linguist. Phon. 2009; 23(1):58-69.

Bunton K. Effects of nasal port area on perception of nasality and measures of nasalance based on computational modeling. Cleft Palate Craniofac J. 2015 Jan;52(1):110-14.

Camargo LOS, Rodrigues CM, Avelar, JA. Oclusão velofaríngea em indivíduos submetidos à nasoendoscopia na clínica de educação para saúde (CEPS). Salusvita. 2001;20(1):35-48.

Capelozza Filho L. Diagnóstico em ortodontia. 1a ed. Maringá: Dental Press Editora; 2004.

Castro-Silva L, Monnazzi MS, Spin-Neto R, Moraes M, Miranda S, Real Gabrielli MF, Pereira-Filho VA. Cone-beam evaluation of pharyngeal airway space in class I, II, and III patients. Oral Surg Oral Med Oral Pathol Oral Radiol. 2015 Dec;120(6):67983.

Chanchareonsook N, Whitehill TL, Samman N. Speech outcome and velopharyngeal function in cleft palate: comparison of Le Fort I maxillary osteotomy and distraction osteogenesis--early results. Cleft Palate Craniofac J. 2007 Jan;44(1):23-32.

Chanchareonsook N, Samman N, Whitehill TL. The effect of cranio-maxillofacial osteotomies and distraction osteogenesis on speech and velopharyngeal status: a critical review. Cleft Palate Craniofac J. 2006 Jul;43(4):477-87.

Chang MK, Sears C, Huang JC, Miller AJ, Kushner HW, Lee JS. Correlation of Airway Volume With Orthognathic Surgical Movement Using Cone-Beam Computed Tomography. J Oral Maxillofac Surg. 2015 Dec;73(12 Suppl):S67-76.

Chua HD, Whitehill TL, Samman N, Cheung LK. Maxillary distraction versus orthognathic surgery in cleft lip and palate patients: effects on speech and velopharyngeal function. Int J Oral Maxillofac Surg. 2010 Jul;39(7):633-40.

D'haeseleer E, Bettens K, De Mets S, De Moor V, Van Lierde K. Normative Data and Dialectical Effects on Nasalance in Flemish Adults. Folia Phoniatr Logop 2015; $67(1): 42-8$. 
Dalston RM, Neiman GS, González-Landa G. Nasometric sensitivity and specificity: a cross-dialect and cross-culture study. Cleft Palate Craniofac J.1993;30(3):285-91.

Dalston RM, Vig PS. Effects of orthognathic surgery on speech: A prospective study. Am J Orthod. 1984;86(4):291-8.

de Felício CM, Medeiros AP, de Oliveira-Melchior M. Validity of the 'protocol of orofacial myofunctional evaluation with scores' for young and adult subjects. J Oral Rehabil. 2012 Oct; 39(10):744-53.

de Freitas CE. Mentoplastia - um importante complemento, não uma solução. In: Araujo A, organizador. Cirurgia Ortognática. São Paulo: Santos; 1999. Capítulo 12, p. 231-74.

Di Ninno CQMS, Krook MIP, Vieira JM, Magalhães LCT, Padovani. Determinação dos valores de nasalância para falantes normais do português brasileiro. Pró-Fono $R$ Atual Cient. 2001;13(1):71-7.

El H, Palamo JM. An airway study of different maxillary and mandibular sagittal positions. Eur J Orthd. 2013 Apr; 35(2):262-70.

El H, Palamo JM. Airway volume for different dentofacial skeletal patterns. Am J Orthod Dentofacial Orthop. 2011 Jun; 139(6):e511-e521.

El-Kassabi RM, Hassan S, Mesallam TA, Malki $\mathrm{KH}$, Farahat M, Alfaris A. Standardization of nasalance scores in normal Saudi speakers. Logoped Phoniatr Vocol. 2015 Jul;40(2):77-85.

Felício CM. Desenvolvimento normal das funções estomatognáticas. In: Fernandes FDM, Mendes BCA, Navas ALPGP, organizadores. Tratado de Fonoaudiologia. 2a ed. São Paulo: Roca; 2009. Capítulo 3, p. 17-27.

Felício CM. Fonoaudiologia aplicada a casos odontológicos: motricidade oral e audiologia. São Paulo: Pancast; 1999. 243 p.

Fletcher SG, Adams LE, Mccutcheon MJ. Cleft palate speech assessment through oral-nasal acoustic measures. In: Bzoch KR, editor. Communicative disorders related to cleft lip and palate. 3rd ed. Boston; 1989, p. 246-57.

Fletcher SG. "Nasalance" vs. listener judgements of nasality. Cleft Palate J. 1976 Jan;13:31-44.

Freitas MR, Alcazar NMPV, Janson G, de Freitas KMS, Henriques JFC. Upper and lower pharyngeal airways in subjects with Class I and Class II malocclusions and different growth patterns. Am J Orthod Dentofacial Orthop. 2006 Dec;130(6):742-5.

Fukushiro AP, Trindade IEK. Nasometric and aerodynamic outcome analysis of pharyngeal flap surgery for the management of velopharyngeal insufficieny. $J$ Craniofac Surg. 2011 Sep;22(5):1647-51. 
Fukushiro AP, Trindade IE. Nasal airway dimensions of adults with cleft lip and palate: differences among cleft types. Cleft Palate Craniofac J. 2005 Jul;42(4):396402.

Garber SR, Speidel TM, Marse G. The effects on speech of surgical premaxillary osteotomy. Am J Orthod. 1981 Jan;79(1):54-62.

Genaro KF, Fukushiro AP, Sugimoto MLFCP. Avaliação e tratamento dos distúrbios da fala. In: Trindade IEK, Silva Filho OG, organizadores. Fissuras labiopalatinas: uma abordagem interdisciplinar. 2a ed São Paulo: Santos; 2007, p. 109-22.

Genaro KF, Yamashita RP, Trindade IEK. Avaliação clínica e instrumental na fissura labiopalatina. In: Fernandes FDM, Mendes BCA, Navas ALPGP, organizadores. Tratado de Fonoaudiologia. 2a ed. São Paulo: Roca; 2009. Capítulo 3, p. 488-503.

Gerek M, Durmaz A, Aydin U, Birkent H, Hidir Y, Tosun F. Relationship between nasal valve changes and nasalance of the voice. Otolaryngol Head Neck Surg. 2012 Jul; 147(1):98-101.

Gildersleeve-Neumann CE, Dalston RM. Nasalance scores in noncleft individuals: why not zero. Cleft Palate Craniofac J. 2001 Mar;38(2):106-11.

Grauer D, Cevidanes LSH, Styner MA, Ackerman JL, Proffit WR. Pharyngeal airway volume and shape from cone-bean computed tomography: relationship to facial morphology. Am J Orthod Dentofacial Orthop. 2009 Dec;136(6):805-14.

Ha S, Cho S-h. Nasalance scores for normal Korean-speaking adults and children: effects of age, vowel context, and stimulus length. Int $\mathrm{J}$ Pediatr Otorhinolaryngol. 2015 Aug;79(8):1235-9.

Haapanen M-L, Kalland M, Heliövaara A, Hukki J, Ranta R. Velopharyngeal function in cleft patients undergoing maxillary advancement. Folia Phoniatr Logop. $1997 ; 49(1): 42-7$.

Harada K, Ishii $Y$, Ishii M, Imaizumi H, Mibu M, Omura K. Effect of maxillary distraction osteogenesis on velopharyngeal function: a pilot study. Oral Surg Med Oral Pathol Oral Radiol Endod. 2002 May;93(5):538-43.

Hassan T, Naini FB, Gill DS. The effects of orthognathic surgery on speech: a review. J Oral Maxillofac Surg. 2007 Dec;65(12):2536-43.

Hong J-S, Oh K-M, Kim B-R, Kim Y-J, Park Y-H. Three-dimensional analysis of pharyngeal airway volume in adults with anterior position of the mandible. Am J Orthod Dentofac Orthop. 2011 Oct;140(4):e161-9.

Ibrahim HM, Reilly S, Kilpatrick N. Normative nasalance scores for the Malay language. Cleft Palate Craniofac J. 2012 Sep;49(5):e61-3. 
Ilg JP. Tratamento ortodôntico-cirúrgico das classes II e III. In: Araujo A, organizador. Cirurgia Ortognática. São Paulo: Santos; 1999. Capítulo 8, p. 107-68.

lqueda APD, Ricz H, Takeshita TK, Reis N, Aguiar-Ricz L. Nasalância e nasalidade da voz traqueosofágica em laringectomizados totais. CoDAS 2013;25(5):469-74.

Jakobsone G, Stenvik A, Espeland L. The effect of maxillary advancement and impaction on the upper airway after bimaxillary surgery to correct Class III malocclusion. Am J Orthod Dentofac Orthop. 2011 Apr;139(4):e369-76.

Janulewicz J, Costello BJ, Buckley MJ, Ford MD, Close J, Gassner R. The effects of Le fort I osteotomies on velopharyngeal and speech functions in cleft patients. J Oral Maxillofac Surg. 2004 Mar;62(3):308-14.

Karabekmez FE, Kleinheinz J, Jung S. Dimensions of velopharyngeal space following maxillary advancement with Le fort I osteotomy compared to Zisser segmental osteotomy: a cephalometric study. Biomed Res Int. 2015; 2015:389605.

Karakoc O, Akcam T, Birkent H, Arslan HH, Gerek M. Nasalance scores for normalspeaking Turkish population. J Craniofac Surg. 2013 Mar;24(2):520-2.

KayPENTAX. Nasometer II Model 6400: Installation, operations and maintenance manual. Lincoln Park, NJ: Kay Elemetrics Corp; 2003.

Kent RD. Speech communication and the speech sciences. San Diego: Singular; 1997, Chapter 1, The speech sciences; p. 01-20.

Kim HK, Yu XM, Cao YJ, Liu XM, Huang ZM. Dialectal and gender differences in nasalance for a Mandarin population. Clin Linguist Phon. 2016;30 (2):119-30.

Kim J-S, Kim JK, Hong S-C, Cho JH. Pharyngeal airway changes after sagittal split ramus osteotomy of the mandible: a comparison between genders. J Oral Maxillofac Surg. 2010 Aug;68(8):1802-6.

Kim $\mathrm{YH}$, Lee $\mathrm{SH}$, Park $\mathrm{CW}$, Cho JH. Nasalance change after sinonasal surgery: analysis of voice after septoturbinoplasty and endoscopic sinus surgery. Am J Rhinol Allergy. 2013 Jan-Feb;27(1):67-70.

Kim Y-J, Hong J-S, Hwang Y-I, Park Y-H. Three-dimensional analysis of pharyngeal airway in preadolescent children with different anteroposterior skeletal patterns. Am J Orthod Dentofacial Orthop. 2010 Mar;137(3):306.e1-306.e11.

Kirjavainen M, Kirjavainen T. Upper airway dimensions in Class II malocclusion. Effects of headgear treatment. Angle Orthod. 2007 Nov;77(6):1046-53.

Ko EW, Figueroa AA, Guyette TW, Polley JW, Law WR. Velopharyngeal changes after maxillary advancement in cleft patients with distraction osteogenesis using a rigid external distraction device: a 1-year cephalometric follow-up. J Craniofac Surg. 1999 Jul;10(4):312-20; discussion 321-2. 
Kochar GD, Chakranarayan A, Kohli S, Kohli VS, Khanna V, Jayan B, Chopra SS, Verma. Effect of surgical mandibular advancement on pharyngeal airway dimensions: a three-dimensional computed tomography study. Int. J. Oral Maxillofac. Surg. 2016; 45:553-9.

Kudo K, Takagi R, Kodama Y, Terao E, Asahito T, Saito I. Evaluation of speech and morphological changes after maxillary advancement for patients with velopharyngeal insufficiency due to repaired cleft palate using a nasometer and lateral cephalogram. J Oral Maxillofac Surg Med Pathol. 2014;26:22-7.

Kummer, AW. Anatomy and physiology: facial, oral, and velopharyngeal structures. Cleft Palate and Craniofacial Anomalies: The Effects on Speech and Resonance, 3rd ed. New Albany, New York: Delmar Cengage Learning; 2013. Chapter 1, p. 2-38.

Kummer, AW. Resonance disorders and velopharyngeal dysfunction. Cleft Palate and Craniofacial Anomalies: The Effects on Speech and Resonance, 3rd ed. New Albany, New York: Delmar Cengage Learning; 2013. Chapter 4, p. 182-224.

Kummer AW, Briggs M, Lee L. The relationship between the characteristics of speech and velopharyngeal gap size. Cleft Palate-Craniofac J. 2003 Nov;40(6):5906.

Kummer AW. Types and causes of velopharyngeal dysfunction. Semin Speech Lang. 2011 May;32(2):150-8.

Lee ASY, Whitehill TL, Ciocca V, Samman N. Acoustic and perceptual analysis of the sibilant sound /s/ before and after orthognathic surgery. J Oral Maxillofac Surg. 2002 Apr;60(4):364-72.

Lee ASY, Whitehill TL, Cioca V. Effect of listener training on perceptual judgement of hypernasality. Clin. Linguist. Phon. 2009; 23(53):319-34.

Lee A, Browne U. Nasalance scores for typical Irish English-speaking adults. Logoped Phoniatr Vocol. 2013 Dec;38(4):167-72.

Lewis KE, Watterson TL, Houghton SM. The influence of listener experience and academic training on ratings of nasality. J Commun Disord. 2002 Jan-Feb;36(1):4958.

Litzaw LL, Dalston RM. The effect of gender upon nasalance scores among normal adult speakers. J Commun Disord. 1992 Mar;25(1):55-64.

Marchesan IQ. Alterações de fala de origem musculoesquelética. In: Ferreira LP, Befi-Lopes DM, Limongi SCO, organizadores. Tratado de Fonoaudiologia. 1a ed. São Paulo: Roca; 2004. Capítulo 25, p.292-303.

Marchesan IQ, Bianchini EMG. A fonoaudiologia e a cirurgia ortognática. In: Araujo A, organizador. Cirurgia Ortognática. São Paulo: Santos; 1999. Capítulo 16, p.35162. 
Marino VCC, Dutka JCR, de Boer G, Cardoso VM, Ramos RG, Bressmann T. Normative Nasalance Scores for Brazilian Portuguese Using New Speech Stimuli. Folia Phoniatr Logop 2016 Feb 5;67(5):238-44.

Marşan G, Hocaoğlu E, Cura N, Emekli U. Nasal profile changes with Le fort I maxillary advancement surgery. Cleft Palate-Craniofac J. 2015 Mar;52(2):152-6.

Martin O, Muelas L, Viñas MJ. Comparative study of nasopharyngeal soft-tissue characteristic in patients with class III malocclusion. Am J Orthod Dentofacial Orthop. 2011 Feb;139(2):245-51.

Mattos CT, Vilani GNL, Sant'Anna EF, Ruellas ACO, Maia LC. Effects of orthognathic surgery on oropharyngeal airway: a meta-analysis. Int J Oral Maxillofac Surg. 2011 Dec;40(12):1347-56.

Maturo DS. Nasalância em indivíduos com deformidades dentofaciais e a influência da cirurgia ortognática [dissertação]. Ribeirão Preto: Universidade de São Paulo, Faculdade de Medicina de Ribeirão Preto; 2017.

Maturo DS, Picinato-Pirola MNC, Ricz LNA, Trawitzki LVV. Nasalância de populações falantes do português brasileiro de dois estados distintos. CoDAS 2017;29(2): e20160041 DOI: 10.1590/2317-1782/20172016041.

McComb RW, Marrinan EM, Nuss RC, LaBrie RA, Mulliken JB, Padwa BL. Predictors of velopharyngeal insufficiency after Le fort I maxillary advancement in patients with cleft palate. J Oral Maxillofac Surg. 2011 Aug;69(8):2226-32.

Miguel HC, Genaro KF, Trindade IEK. Avaliação perceptiva e instrumental da função velofaríngea na fissura de palato submucosa assintomática. Pró-Fono $\mathrm{R}$ Atual Cient. 2007 Jan-Feb;19(1):105-12.

Mishima K, Sugii U, Yamada T, Imura H, Sugahara T. Dialectal and gender differences in nasalance scores in Japanese population. J Craniomaxillofac Surg. 2008 Jan; 36(1):8-10.

Nascimento KSG. Desempenho funcional da língua na deformidade dentofacial [dissertação]. Ribeirão Preto: Universidade de São Paulo, Faculdade de Medicina de Ribeirão Preto; 2015.

Narece IL. Nasalância de crianças com fissura labiopalatina e nasalidade de fala normal: uma comparação dos dialetos mineiro e paulista [dissertação]. São Carlos: Universidade de São Paulo, Bioengenharia; 2007.

Niemeyer TC, Gomes AOC, Fukushiro AP, Genaro KF. Speech resonance in orthognathic surgery in subjects with cleft lip and palate. J Appl Oral Sci. 2005;13(3):232-6. 
Nieminen P, Löppönen H, Väyrynen M, Tervonen A, Tolonen U. Nasalance scores in snoring children with obstructive symptoms. Int J Pediatr Otorhinolaryngol. 2000 Jan 30;52(1):53-60.

O'Gara M, Wilson K. The effects of maxillofacial surgery on speech and velopharyngeal function. Clin Plastic Surg. 2007 Jul;34(3):395-402.

Okalidou A, Karathanasi A, Grigoraki E. Nasalance norms in Greek adults. Clin Linguist Phon. 2011 Aug;25(8):671-88.

Park M, Baek WS, Lee E, Koh KS, Kim B-k, Baek R. Nasalance scores for normal Korean-speaking adults and children. J Plast Reconstr Aesthet Surg. 2014 Feb;67(2):173-7.

Park S-B, Kim Y-L, Son W-S, Hwang D-S, Cho B-H. Cone-bean computed tomography evaluation short- and long-term airway change and stability after orthognathic surgery in patients with Class III skeletal deformities: Bimaxillary surgery and mandibular setback surgery. Int J Oral Maxillofac Surg. 2012 Jan;41(1):87-93.

Pegoraro-Krook MI, Dutka JCR, Magalhães LCT, Feniman MR. Intervenção fonoaudiológica na fissura palatina. In: Ferreira LP, Befi-Lopes DM, Limongi SCO, organizadores. Tratado de Fonoaudiologia. 1a ed. São Paulo: Roca; 2004. Cap. 35, p.439-455.

Pegoraro-Krook MI, Dutka-Souza JCR, Williams WN, Magalhães LCT, Rossetto PC, Riski JE. Effect of nasal decongestion on nasalance measures. Cleft PalateCraniofac J. 2006 May;43(3):289-94.

Pereira VJ, Sell D, Tuomainen J. Effect of maxillary osteotomy on speech in cleft lip and palate: perceptual outcomes of velopharyngeal function. Int $\mathrm{J}$ Lang Commun Disord. 2013 Nov-Dec;48(6):640-50.

Picinato-Pirola MNC, Mello-Filho FV, Trawitzki LVV. Masticatory efficiency in class II and class III dentofacial deformities. Int J Oral Maxillofac Surg. 2012 Jul;41(7):830-4.

Proffit WR, White Jr RP. Who needs surgical-orthodontic treatment? Int J Adult Orthod Orthog Surg. 1990;5(2):81-9.

Raimundo GM. Medidas de nasalância em crianças com fissura labiopalatina e fala normal [dissertação]. São Carlos: Universidade de São Paulo, Escola de Engenharia de São Carlos - Faculdade de Medicina de Ribeirão Preto - Instituto de Química de São Carlos; 2007.

Ribas MO, Reis LFG, França BHS, de Lima AAS. Cirurgia ortognática: orientações legais aos ortodontistas e cirurgiões bucofaciais. R Dental Press Ortodon Ortop Fac. 2005 Nov-Dec;10(6):75-83.

Rochet AP, Rochet BL, Sovis EA, Mielke DL. [Characteristics of nasalance in speakers of western Canadian English and French]. Revue D'Orthophonie et D’Audiologie. 1998;22(2):94-102. French. 
Ruscello DM, Tekieli ME, Jakomis T, Cook L, Sickels. The effects of orthognathic surgery on speech production. Am J Orthod. 1986 Mar;89(3):237-41.

Scarmagnani $\mathrm{RH}$, Oliveira AC, Fukushiro AP, Salgado $\mathrm{MH}$, Trindade IE, Yamashita RP. O impacto da concordância entre avaliadores no julgamento perceptivo da nasalidade da fala. Codas. 2014 Set-Out;26(5):357-9.

Scarsellone JM, Rochet AP, Wolfaardt JF. The influence of dentures on nasalance values in speech. Cleft Palate-Craniofac J. 1999 Jan;36(1):51-6.

Seaver EJ, Dalston RM, Leeper HA, Adams LE. A study of nasometric values for normal resonance. J Speech Hear Res. 1991 Aug;34(4):715-21.

Smedberg E, Neovius E, Lohmander A. Impact of maxillary advancement on speech and velopharyngeal function in patients with cleft lip and palate. Cleft PalateCraniofac J. 2014 May;51(3):334-43.

Soneghet R, Santos RP, Behlau M, Habermann, Friedrich G, Stammberger H. Nasalance changes after functional endoscopic sinus surgery. J Voice. 2002 Sep;16(3):392-7.

Suguimoto MLCP, Pegoraro-Krook MI. Avaliação nasométrica em adultos normais falantes do Português Brasileiro. Pró-Fono. 1996;8(1):7-12.

Sweeney T, Sell D, O'regan M. Nasalance scores for normal-speaking Irish children. Cleft Palate-Craniofac J. 2004;41(2):168-74.

Tanigute CC. Desenvolvimento das funções estomatognáticas. In: Marchesan IQ, editor. Fundamentos em fonoaudiologia: aspectos clínicos da motricidade oral. $2 a$ ed. Rio de Janeiro: Guanabara-Koogan; 2005. Capítulo 1, p. 1-9.

Thorp EB, Vimik BT, Stepp CE. Comparison of nasal acceleration and nasalance across vowels. J Speech Lang Hear Res. 2013 Oct;56(5):1476-84.

Trawitzki LVV. Fonoaudiologia nas deformidades dentofaciais junto à equipe de cirurgia ortognática. In: Felício CM, Trawitzki LVV, organizadoras. Interfaces da medicina, odontologia e fonoaudiologia no complexo cérvico-craniofacial. Barueri: Pró-Fono; 2009. Capítulo 13, p. 267-88.

Trawitzki LVV, Borges CGP, Grechi TH. Fonoaudiologia em casos de cirurgia ortognática. In: Berretin-Felix G, Alves GAS, Silva LK, Rosa RR, Silva HJ, organizadores. Interfaces e tecnologias em motricidade orofacial. São José dos Campos: Pulso editorial; 2016. Capítulo 4, p. 59-70.

Trawitzki LVV, Puppin-Rontani RM, Felício CM, Vitti M, Matsumoto MAN. Investigação eletromigráfica dos músculos masseter e temporal durante a mastigação em crianças com diferentes tendências de crescimento facial. R Soc Bras Fonoaudiol. 2000;7(7):54-8. 
Trindade IEK, Yamashita RP, Suguimoto RM, Mazzotini R, Trindade Jr AS. Effects of orthognathic surgery on speech and breathing of subjects with cleft lip and palate: acoustic and aerodynamic assessment. Cleft Palate J. 2003 Jan;40 (1):54-6.

Trindade IEK, Genaro KF, Dalston RM. Nasalance scores of normal Brazilian Portuguese speakers. Braz J Dysmorphol Speech Hear Disord. 1997;1:23-34.

Trindade IEK, Yamashita RP, Gonçalves CGAB. Diagnóstico instrumental da disfunção velofaríngea. In: Trindade IEK, Silva Filho OG, organizadores. Fissuras labiopalatinas: uma abordagem interdisciplinar. 2a ed São Paulo: Santos; 2007, p. 123-143.

Tucker MR. Correção das deformidades dentofaciais. In: Peterson LJ, editor. Cirurgia oral e maxilofacial contemporânea. 3a ed. Rio de Janeiro: Guanabara Koogan; 2000. Capítulo 26, p. 600-38.

Tuzuner A, Demirci S, Akkoc A, Arslan E, Arslan N. Nasalance scores in pediatric patients after adenoidectomy. Int J Pediatr Otorhinolaryngol. 2014 Apr;78(4):610-13.

Vallino LD. Speech, velopharyngeal function, and hearing before and after orthognathic surgery. J Oral Maxillofac Surg.1990 Dec;48(12):1274-81.

Van Lierde KM, Schepers S, Timmermans L, Verhoye I, Van Cauwenberge P. The impact of mandibular advancement on articulation, resonance and voice characteristics in Flemish speaking adults: a pilot study. Int J Oral Maxillofac Surg. 2006 Feb;35(2):137-44.

Van Lierde KM, Wuyts FL, Bonte K. The Nasality Severity Index: An Objective Measure of Hypernasality Based on a Multiparameter Approach. Folia Phoniatr Logop. 2007;59(1):31-8.

Van Lierde KM, Wuyts FL, De Bodt M, Van Cauwenberge P. Nasometric values for normal nasal resonance in the speech of young Flemish adults. Cleft Palate Craniofac J. 2001 Mar;38(2):112-18.

Van Lierde KM, Wuyts FL, De Bodt M, Van Cauwenberge P. Age-related patterns of nasal resonance in normal Flemish children and young adults. Scand J Plast Reconstr Surg Hand Surg. 2003;37(6):344-50.

Van Lierde KM, Van Borsel J, Cardinael A, Reeckmans S, Bonte K. The impact of vocal intensity and pitch modulation on nasalance scores: a pilot study. Folia Phoniatr Logop. 2011;63(1):21-6.

Ward EC, McAuliffe M, Holmes SK, Lynham A, Monsour F. Impact of malocclusion and orthognathic reconstruction surgery on resonance and articulatory function: an examination of variability in five cases. $\mathrm{Br} J$ Oral Maxillofac Surg. 2002 Out;40(5):410-17. 
Williams BJ, Isom A, Laureano Filho JR, O'Ryan FS. Nasal airway function after maxillary surgery: a prospective cohort study using the nasal obstruction symptom evaluation scale. J Oral Maxillofac Surg. 2013 Feb;71(2):343-50.

Xavier SP, Ribeiro HT, Pedrosa-Júnior WF. Cirurgia ortognática: noções de técnicas cirúrgicas. In: Felício CM, Trawitzki LVV, organizadoras. Interfaces da medicina, odontologia e fonoaudiologia no complexo cérvico-craniofacial. Barueri: Pró-Fono; 2009. Capítulo 12, p. 237-65.

Zemann W, Feichtinger M, Santler G, Kärcher H. [Effects of Le-Fort-I-Osteotomy on nasalance scores]. Mund Kiefer Gesichtschir. 2006 Jul;10(4):221-8. German. 


\section{APÊNDICE A - Termo de Consentimento Livre e Esclarecido - Grupo Estudo}

\author{
TERMO DE CONSENTIMENTO LIVRE E ESCLARECIDO
}

Eu, Denise Silva Maturo, fonoaudióloga e pesquisadora responsável, 0 convido a participar de uma pesquisa que tem como objetivo verificar se indivíduos com deformidades dentofaciais apresentam o mesmo padräo de nasalància (sotaque nasal) e se a realização da cirurgia ortognática causa alguma mudança nessa nasalância.

Antes da cirurgia, será realizada uma entrevista, perguntando dados referentes à sua identificaçäo, cidade de origem, problemas para respirar e para ouvir, se fez alguma cinurgia na boca e na face, se teve traumas na face, dificuldade para se alimentar e problemas na voz. Em seguida vocé será submetido a uma avaliaçăo clínica da face e da boca, na qual será analisado como ficam os seus lábios (se fechados ou nảo), a posiçäo da sua língua, o formato do "céu da boca", da "campainha" (chamada de véu palatino) e das amigdalas e a mobilidade da regiäo da campainha; uma avaliação da respiraçäo, para identificar uma respiração pelo nariz, pela boca ou por ambos e a quantidade de ar que sai pelo nariz, e por fim, a investigaçäo de como voce fala, analisando se tem algumas alteraçoes. Para avaliar a nasalància, você deverá colocar uma espécie de capacete, equipado com dois microfones, um direcionado à boca e outro ao nariz, separados por uma placa horizontal. Durante o exame vocé irá ler um total de 10 frases, que seräo apresentadas na tela de um computador, sendo assim registrado o valor de nasaläncia da fala em porcentagem (\%). 0 exame é rápido e não causará dor ou desconforto e, enquanto o realiza vocé permanecerá sentado em uma cadeira confortável no Laboratório de Fonoaudiologia da Faculdade de Medicina de Ribeiräo Preto da Universidade de São Paulo (casa 18) situado na Rua das Paineiras, da faculdade acima citada. 0 exame será feito um dia antes da cirurgia.

Após um mês da realização da cirurgia, vocé passará por uma nova avaliaçäo clínica da face e da boca, avaliaçäo da respiraçäo, investigaçäo da fala e avaliaçäo da nasalância. Esses procedimentos seräo repetidos nos periodos de 3 e 6 meses, após a cirurgia.

A pesquisadora se compromete a prestar assistencia integral no decorrer da pesquisa se algum problema decorrer desta.

0 ressarcimento de eventuais despesas, bem como a indenizaçäo, a título de cobertura material, para reparaçäo de danos imediatos ou tardios, decorrentes da sua participaçäo na pesquisa, seräo feitos por mim, näo cabendo ao Hospital das Clínicas
Eu manterei sigilo sobre a sua identidade. Como sua participaçäo é voluntária você tem o direito de interrompê-la em qualquer momento, sem sorrer penalizaçöes e sem comprometer seus atendimentos no Ambulatório de Otorrinolaringologia.

Concordando em participar da pesquisa voluntariamente você assinará 0 Temo de Consentimento Live e Esclarecido, no qual constam os dados do Pesquisador Responsável, caso necessite de maiores informaçōes, ou por qualquer outra necessidade.

Eu, RG assino este Termo de Consentimento com a finalidade de autorizar minha participaçäo como sujeito da pesquisa intitulada "NASALÂNCIA EM INDIVÍDUOS COM DEFORMIDADES DENTOFACIAIS E A INFLUÊNCIA DA CIRURGIA ORTOGNÁTICA" sob responsabilidade da aluna de mestrado Denise Silva Maturo e afirmo que foram dadas todas as explicaçōes necessárias para eu tomar essa decisão de livre e espontânea vontade. Recebi as seguintes informaçöes, junto a uma cópia desse Termo de Consentimento:

Dados do Pesquisador responsível e Orientadora para eventuais necessidades: Fga. Denise Silva Maturo e Profa. Dra. Luciana Vitaliano Voi Trawitzki

End: Rua das Paineiras, 18, Campus Universitánio da USP, Riberäo Preto, Cep: 14049.800 Tel: (18) 36020502

Dados do Comitê de Etica em Pesquisa do HC

End: Avenida Banderantes, 3800, Riberiåo Preto, Cepp: 14048.800

Tel: (16) $3602-2228$

E-mail: cepQherp.tmrp.usp.br

Ribeiräo Preto, ........ de de 201 . 


\section{APÊNDICE B - Termo de Consentimento Livre e Esclarecido - Grupo Controle}

\author{
TERMO DE CONSENTIMENTO LIVRE E ESCLARECIDO \\ Grupo Controle
}

Eu, Denise SIva Maturo, fonoaudlogga e pesquisadora responsavel, o convido a participar de uma pesquisa que tem como objetivo verticar se indlviduos com detomidades dentofaclals apresentiam o mesmo padrfo de nassalthncla (sotaque nasal) e se a real|zaç̧o da cinurgla otognatlca causa alguma mudança nessa nasalsncla.

Serd reallzada umia entrevsta, perguntando dados referentes a sua Identiticaçfo, cidade de orlgem, problemas para respirar e para ouvit, se fez aguma cirurgla na boca e na face, se teve traumas na tace, ofeculdade para se almentar e

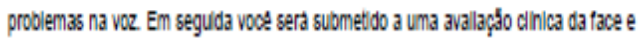
da boca, na qual sera anal|sado como fcam os seus lablos (se fechados ou nab), a posiģ̧o da sua Ingua, o formatio do "ceu da bocas", da "campanhas" (chamado de veu pazatho) e das amigdalas e a mobilloade da reglfo da campainha; uma avalaça da

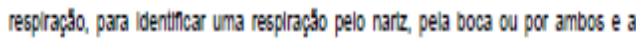
quaritloade de ar que sal pelo nariz, e por fim, a Investigagfo de como voce tala, anal|sando se tem algumas atterą̧es. Para avallar a nasalancla, voce devera colocar uma especcle de capacte, equpasdo com dols microtones, un drececonaso a boca e outro as narz, separados por uma placa hortzontal. Durante o exame voce ira ler um total de 10 trases, que serdo apresentiadas na tela de um computador, sendo assim regstrado o valor de nasalancla da fala em porcentagem (\%), 0 exame e rapido e nło causara dor ou desconforto e, enquanto o realza voce permanecera sentado em uma cadelra contoritvel no Laboratorlo de Fonoaudologla da Facudade de Medlcha de Riberido Preto da Unversidade de Sto Paulo (casa 18) stuaco na Rua das Palnelras, da facudade acma ctada. 0 exame sera fetto en um dla.

A pesqulsadora se compromete a prestar assistencla integral no decorrer da pesquisa se algum proolema deconrer desta

O rescarcimentio de eventuas despesss, bem como a nderizaçoto, a titulo de cobertura materala, para reparaç5o de danos imedlatos ou tardloc, deconrentes da sua

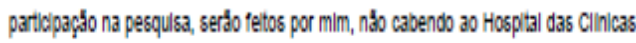
de Riberdo Preto, qualquer responsabllidade quarito a0s referilios pagamentos.

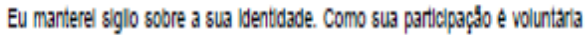
voce tem 0 dretto de interrompe-la en qualquer monnentio, sem sofrer penal zaçeses.

Concordando em particlpar da pesquisa volunitarlamente voce assinara 0 Termo de Consentmento Lure e Escarecido, no qual consiam os dasocs do
Pesquisador Responsavel, caso necessite de malores informaçes, ou por quaiquer ourta necascidade.

Eu, RG assino

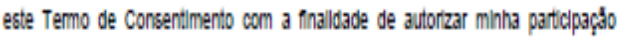
como suletio da pesqulsa Intiluada "NASALANCIA EM INDIVIDUOS COM DEFORMIDADES DENTOFACIAIS E A INFLUENCIA DA CIRURGIA ORTOGNATICA' sob responsabllidade da aluna de mestrado Denlise Slva Maturo e afrmo que foram dasass todas as explicaşes necessarlas para eu tomar essa

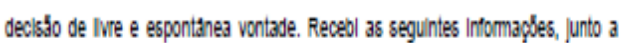
uma copla desse Termo de Consentimentio:

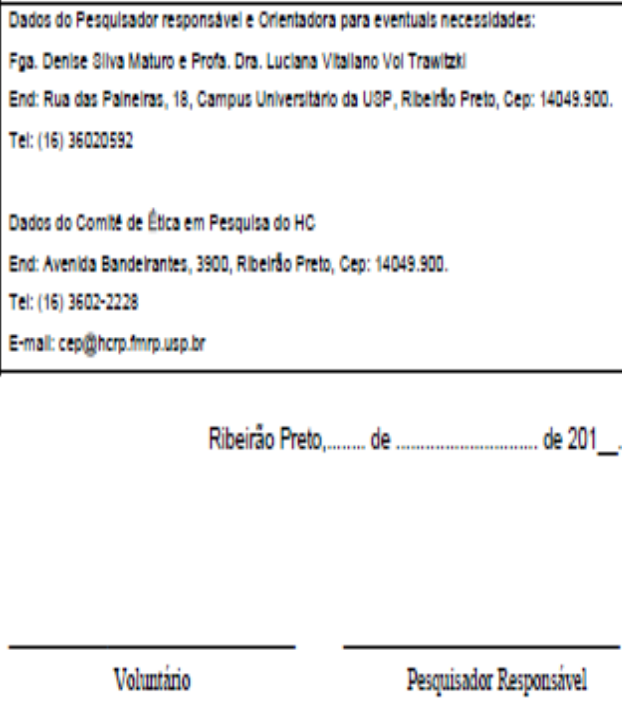

Riberiåo Preto, ........ de de 201 Pesquicisador Responsivive] 
APÊNDICE C - Entrevista desenvolvida para a pesquisa

\section{Identincaçăo}

Nome:

Data de nascimento: Idade atual:

Sexo:

Naturalidade:

Nacionalidade:

Nivel de escolaridade: Profissão:

Rua: Número: Apto:

Bairro: Cidade: CEP:

Telefone/ recado:

\section{Questionário}

1. Há quanto tempo você mora nessa região?

2. Você já morou em outro estado/região do país? ( ) não ( ) sim...Qual(is) e por quanto tempo (ordem cronológica)

3. Possui algum problema respiratório?
( ) nã̀o
( ) sim... Qual?

4. Jả fez alguma cirurgia no nariz?

( ) nåo ( ) sim... Qual?

5. Já fez alguma cirurgia na garganta?

( ) não ( ) sim... Qual?

6. Já fez alguma cirurgia na boca?
( ) não
( ) sim... Qual?

7. Já sofreu algum trauma na face?
( ) não
( ) sim... Qual?

8. Apresenta algum problema para se alimentar (engasgos, refluxo nasal)?
( ) não
( ) sim... Qual?

9. Possui algum problema vocal?
( ) não
( ) sim... Qual?

10. Já fez algum tratamento para a voz?
( ) não
( ) sim... Qual?

11. Apresenta algum problema auditivo?

( ) nå ( ) sim... Qual? 
APÊNDICE D - Complemento da avaliação miofuncional desenvolvido para a pesquisa

\section{1-Morfologia da úvuiz:}
( ) sem alteraçbes
( ) bifida
( ) sulcada
( ) hipoplásica

2-Amigdalas Palatinas:
( ) sem alteraçōes
( ) ausente
( ) toca na linha média
( ) além dos pilares, porém sem tocar a linha média

3- Mobilidade do véu palatino:
( ) presente
( ) ausente
( ) desvia para a dircita
( ) desvia para a esquerda

4- Obstrução Nasal (espelho milimetrado de Altmann):

\section{Direita}

() Sem obstrução

( ) Parcialmente obstruido

() Totalmente obstruido

\section{Esquerda}

() Sem obstrução

( ) Parcialmente obstruido

( ) Totalmente obstruido

\section{5-Prejuizo na Inteligibilidade de Fala:}
() ausente
( ) leve
( ) leve para moderado
( ) moderado
( ) moderado para severo
( ) severo
( ) ininteligivel

\section{6-Ressonância:}

( ) Equilibrada
Hipernasalidade: ( ) leve
() moderada
() severa
Hiponasalidade: ( ) leve
( ) moderada
( ) severa

7-Teste de Emissão de Ar Nasal:
( ) sopro
( ) sopro com a língua para fora
() $/ 6$
( ) $/ \mathrm{s} /$
( ) $/ S /$
( ) papai
( ) tatu
() caqui
() babả
() dedo
() Guga
() fafá
( ) saci
( ) Xuxa
( ) vovó
( ) Zezé
() Gigi 
ANEXO A - Aprovação pelo Comitê de Ética

HOSPITAL DAS CLÍNICAS DA FACULDADE DE MEDICINA DE RIBEIRÃO PRETO DA UNIVERSIDADE DE SÃO PAULO

Ribeirão Preto, 10 de outubro de 2012

Ofício $\mathrm{n}^{\circ} 3550 / 2012$

CEP/MGV

PROCESSO HCRP $\mathrm{n}^{\circ} 10870 / 2012$

Prezadas Pesquisadoras,

O trabalho intitulado "NASALÂNCIA EM INDTVIDUOS COM DEFORMIDADES DENTOFACIAIS E A INTLUÊNCIA DA CIRURGIA ORTOGNÁTICA", foi analisado pelo Comitê de Ética em Pesquisa, em sua $355^{a}$ Reunião Ordinária realizada em 08/10/2012, e enquadrado na categoria: APROVADO, bem como o Termo de Consentimento Livre e Esclarecido.

Este Comitê segue integralmente a Conferência Internacional de Harmonização de Boas Práticas Clinicas (IGH-GCP), bem como a Resolução $n^{\circ}$ 196/96 CNS/MS.

Lembramos que devem ser apresentados a este CEP, o Relatório
Parcial e o Relatório Final da pesquisa. De acordo com Carta Circular $n^{\circ}$ O03/2011/CONEP/CNS, datada de 21/03/2011, o sujeito de pesquisa ou seu representante, quando for o caso, deverá rubricar todas as folhas do Termo de Consentimento Livre e Esclarecido - TCLE - apondo sua assinatura na última do referido Termo; o pesquisador responsável deverá da mesma forma, rubricar todas as folhas do Termo de Consentimento Livre e Esclarecido - TCLE - apondo sua assinatura na última página do referido Termo.

Atenciosamente.

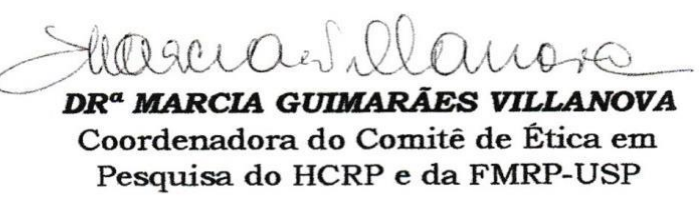

Ilustrissimas Senhoras

DENISE SILVA MATURO

PROF $^{a}$ DR $^{\text {a }}$ LUCIANA VITALIANO VOI TRAWITZKI (Orientadora)

Depto. de Oftalmologia, Otorrinolaringologia e Cirurgia de Cabeça e Pescoço 
ANEXO B - Itens utilizados do Protocolo de Avaliação Miofuncional Orofacial com Escores - AMIOFE (de Felício; Medeiros; de Oliveira-Melchior, 2012)

APARÊNCIA E CONDIÇÄO POSTURAL/POSIÇÃO

\begin{tabular}{|c|c|c|}
\hline \multicolumn{2}{|l|}{ Condiçăo Postural dos Lábios } & Escores \\
\hline Oclusão normal dos lábios & Normal & (3) \\
\hline Oclusão dos lábios com Tensão & $\begin{array}{l}\text { Atividade aumentada dos lábios e Mm. } \\
\text { Mentalis }\end{array}$ & (2) \\
\hline Ausência de oclusão labial & Disfunção leve & (2) \\
\hline & Disfunção severa & (1) \\
\hline
\end{tabular}

\begin{tabular}{|l|l|l|}
\hline \multicolumn{2}{|l|}{ Posição da Língua } & Escores \\
\hline Contida na cavidade oral & Normal & $(3)$ \\
\hline Interposta aos arcos dentários & Adaptação ou disfunção & $(2)$ \\
\cline { 2 - 3 } & Protruída em excesso & $(1)$ \\
\hline Resultado do sujeito avaliado & & Escores \\
\hline Aparência do Palato Duro & & $(3)$ \\
\hline & Normal & $(2)$ \\
\hline Largura diminuída (estreito) & Leve & $(1)$ \\
\cline { 2 - 3 } & Severo & \\
\hline Resultado do sujeito avaliado & & \\
\hline
\end{tabular}

FUNÇöES

\begin{tabular}{|l|l|l|}
\hline Respiração & Escores \\
\hline Respiração nasal & Normal & $(3)$ \\
\hline Respiração oronasal & Leve & $(2)$ \\
\cline { 2 - 3 } & Severa & $(1)$ \\
\hline Resultado do sujeito avaliado & \\
\hline
\end{tabular}


ANEXO C - Frases para avaliação nasométrica (Trindade; Genaro; Dalston, 1997)

\section{Texto Oral [ZOO-BR]}

Papai caiu da escada.

Fábio pegou o gelo.

O palhaço chutou a bola.

Tereza fez pastel.

A árvore dá frutos e flores.

\section{Texto Nasal [NASAL2-BR]}

Aninha ama o alemão.

O anão melou a mão.

A lenha molhou a lama.

O leão unhou o ninho.

O nenê mamou na mamãe. 